\title{
The Use of Finite Polynomial Rings in the Factorization of the General Polynomial
}

\author{
Daniel B. Lloyd*
}

(May 26, 1965)

\begin{abstract}
The problem of decomposing the general polynomial over the integers into its prime polynomial factors is solved by the use of congruential functions.

The following principles are used:

I. If a polynomial $P$ is reducible into rational integral factors, then $P_{m}$ is similarly reducible in $J_{m}$, where $P_{m}$ is the polynomial $P$ with its coefficients reduced modulo $m$ ( $m$ a prime).

II. The factors of $P_{m}$ are congruent modulo $m$ to the factors of $P$. of I.)

III. If $P_{m}$ is irreducible in $J_{m}$, then $P$ is irreducible over the rational integers. (Contrapositive

Accompanying tables are provided which give the factors of all congruential polynomials over the ring of integers modulo $2,3,5$, up through degrees 11,11 , and 8 , respectively.
\end{abstract}

\section{Introduction}

\section{Foreword}

The first set of tables of this kind were designed several years ago by the author at the University of Oklahoma. At their computing center they were computed through degree 6 for moduli 2, 3, 5, and 7, under the direction of R. V. Andree, and C. E. Maudlin, programmer. Based on those tables, the present tables were designed with a more concise format. The latter were computed in 1964 through the courtesy of the University of Maryland Computer Science Center, Werner C. Rheinboldt, Director, and Harry Remmers, Jr., programmer. They were programmed in Fortran IV on a No. 7090 IBM machine. The actual computing time on the machine was as follows:

\begin{tabular}{|c|c|c|}
\hline Modulus 2, degrees 1-11 & $0 \mathrm{~h}$ & $1.6 \mathrm{~m}$ \\
\hline Modulus 3, degrees 1-11 & $2 \mathrm{~h}$ & $7.6 \mathrm{~m}$ \\
\hline Modulus 5, degrees 1-8 & $6 \mathrm{~h}$ & $20.0 \mathrm{~m}$ \\
\hline
\end{tabular}

Due to the extensive size of the tables for moduli 3 and 5 , only the modulus 2 tables are included in full in this paper. However, sample extracts from the moduli 3 and 5 tables are given to show their use in conjunction with the illustrative examples given below.

These factor tables have been computed for moduli 2 and 3 through degree 11 for modulus 5 through degree 8 , and modulus 7 through degree 6 . These have not as yet been published but persons desiring them may contact the author in Washington, D.C.

No other factor tables for all polynomials, reducible and irreducible have heretofore been published. Previously there have been published tables of irreducible polynomials, notably the following:

*Present address: Catholic University, Washington, D.C. (a) Church, Randolph. Tables of Irreducible Polynomials for the First Four Prime Moduli. Annals of Mathematics 36, 198-209. (1935).

They include:

Mod 2, Degrees 1-11

Mod 3, Degrees 1-7

Mod 5, Degrees 1-5

Mod 7, Degrees 1-4.

(b) Marsh, R. W. Table of Irreducible Polynomials Over GF(2) Through Degree 19. National Security Administration, W ashington, D.C. (1957).

(c) Peterson, W. W. "Error-Correcting Codes." M. I. T. (1961). An appendix, pp. 251-270, lists the irreducible polynomials, degrees 2-16; for degrees 17-34 are listed only certain primitive polynomials belonging to various possible exponents.

In the publication of this present paper the author appreciates the helpful suggestions of Morris Newman and John E. Carpenter, of the Bureau of Standards Staff, and of John W. Wrench, Jr., of the David Taylor Model Basin.

\section{Description of the Factor Tables}

The tables list consecutively the monic congruential polynomials with respect to moduli 2,3 , and 5 . Opposite each polynomial a prime factor of least degree, and the resulting quotient, are given. For example, in modulus 3 , degree 6 :

$$
x^{6}+2 x^{5}+2 x^{3}+x^{2}+x+1=\left(x^{2}+2 x+2\right)\left(x^{4}+x^{2}+2\right) .
$$

The second factor may or may not be prime, and would require further investigation.

However, to produce a concise format, only the detached coefficients are printed. These are further abbreviated by a condensed system of numeration, octic for modulus 2, and a modified form for mod- 


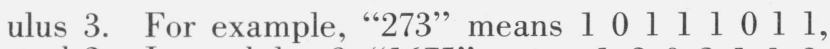

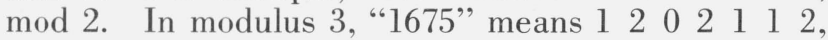
wherein each two digits condense ternary-wise into one. A condensed notation is not used in modulus 5 .

A further shortening of the tables is effected by omitting reciprocal polynomials. Every polynomial has a "reciprocal," formed by writing the coefficients in the reverse order. This reciprocal has factors which are the reciprocals of those of the original, and hence need not be listed.

Thus, in degree 4 , modulus 3 , $1111112=\left(\begin{array}{ll}1 & 2\end{array}\right)$ (1 $\left.\begin{array}{llll}1 & 2 & 0 & 1\end{array}\right)$, while its reciprocal (not listed), $1 \begin{array}{lllll}1 & 2 & 2 & 2 & 2\end{array}$ $=\left(\begin{array}{lllll}1 & 2\end{array}\right)\left(\begin{array}{llll}1 & 0 & 2 & 1\end{array}\right)$. And, in modulus 5, $1113 \quad 3 \quad 1 \quad 2 \quad 1$ $=\left(\begin{array}{lll}1 & 3 & 4\end{array}\right)\left(\begin{array}{llll}1 & 3 & 0 & 4\end{array}\right)$. While its reciprocal (not listed),

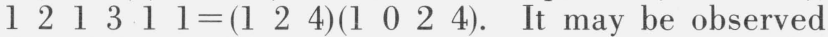
that $\left(\begin{array}{ll}1 & 2\end{array}\right)$ is its own reciprocal in $\bmod 3$, while in modulus 5 coefficients $(1,4),(2,3),(0)$ are "reciprocal" pairs.

The second polynomial of each of these pairs is omitted from the table, being of greater "height" of the pair. In using the tables one must thus seek the lesser of the pair, reciprocating and reversing the coefficients as necessary. As only monic polynomials are listed, it will be necessary to reduce coefficients in this process, unless the constant term is unity. The illustrative problems below clarify this procedure.

Irreducible polynomials are listed without factors.

\section{Present Status of the Factoring Problem}

The problem of decomposing the general rational polynomial into its prime polynomial factors has vexed mathematicians for a long time. It has never been completely solved except by tedious empirical methods. Typical of these are the methods advanced by Kronecker [8], ${ }^{3}$ Runge [17], Mandl [11], Glenn [6], and Frumveller [5], but the computational procedures involved in these were lengthy and onerous. A critique of these methods is given in [9] and need not be repeated here.

The related problem of determining the reducibility or irreducibility of a polynomial has also attracted much attention. Success in this effort has been confined to special cases only. Some necessary conditions and some sufficient ones have been found, but no conditions that are both necessary and sufficient. A necessary and sufficient condition for reducibility of the quartic was found by Varnhorn [20] in 1939, and by the author [9] for the quintic in 1940. Their methods also led to the actual determination of the factors. But no absolute criterion for the reducibility of the general polynomial exists in the literature.

A number of writers have presented criteria for the irreducibility of certain classes of polynomials. Among these are Schoenemann [18], Eisenstein [4], Königsberger [7], Netto [12], Bauer [1], Perron [14], Dumas [3], Ore [13], Stäckel [19], and Polya and Szego [16]. It will not be necessary to outline their work here as such a historical survey was made by Dorwart [2] in 1935. Whereas the above schemes are helpful for certain kinds of polynomials, actually they are applicable to only a small percentage of cases.

\footnotetext{
${ }^{3}$ Figures in brackets indicate the literature references at the end of this paper
}

From the above survey it is clear that a broader attack on the related problems of reducibility and decomposition of the general polynomial is highly desirable.

\section{The Theory of the Proposed Method}

We shall consider the general polynomial over $J$, the ring of rational integers. Further, without loss of generality, we limit our discussion to monic polynomials (leading coefficient unity), for a simple transformation will render monic any given polynomial.

We establish the correspondence $a \rightarrow a_{m}$, which carries each integer $a$ into its residue class modulo $m$, where $m$ is a prime. This is a homomorphism of the ring of rational integers to the finite ring of integers, $J_{m}$. We then consider the general polynomial $P$ with rational integral coefficients. The correspondence $P \rightarrow P_{m}$ carries $P$ into a finite ring of polynomials, with coefficients in $J_{m}$. We present the following three theorems with proof below (cf. reference [10]):

I. If a polynomial $P$ is reducible into rational integral factors, then $P_{m}$ is similarly reducible in $J_{m}$, where $P_{m}$ is the polynomial $P$ with its coefficients reduced modulo $m,(m$ a prime).

II. The factors of $\boldsymbol{P}_{m}$ are congruent modulo $m$ to the factors of $P$.

III. If $P_{m}$ is irreducible in $J_{m}$, then $P$ is irreducible over the rational integers. (Contrapositive of I.)

Proof:

Let

$$
P=\sum_{i=0}^{n} \quad C_{i} x^{i}
$$

and the corresponding reduced polynomial

$$
P_{m}=\sum_{i=0}^{n} c_{i} x^{i}
$$

where each $C_{i}=m q_{i}+c_{i}$

$$
0 \leqslant c_{i}<m .
$$

Assume $P=Q \cdot R$ the product of two rational integral polynomials, where $Q=\sum_{i=0}^{k} M_{i} x^{i}$ and $R=\sum_{i=0}^{n-k} N_{i} x^{i}$ $0<k<n$.

And their corresponding reduced polynomials

$$
Q_{m}=\sum_{i=0}^{k} m_{i} x^{i} \text { and } R_{m}=\sum_{i=0}^{n-k} n_{i} x^{i}
$$

when $M_{i}=m l_{i}+m_{i}, N_{i}=m t_{i}+n_{i}, 0 \leqslant m_{i}, n_{i}<m$.

Then

$$
\begin{gathered}
P=\sum_{i=0}^{n}\left(m q_{i}+c_{i}\right) x^{i} . \\
P=\sum_{i=0}^{k} M_{i} x^{i} \sum_{i=0}^{n-k} N_{i} x^{i} \\
=\sum_{i=0}^{k}\left(m l_{i}+m_{i}\right) x^{i} \sum_{i=0}^{n-k}\left(m t_{i}+n_{i}\right) x^{i} .
\end{gathered}
$$


Noting the identity of (1) and (2), and by reducing coefficients, modulo $m$, we have the congruence:

$$
\sum_{i=0}^{n} c_{i} x^{i}=\left(\sum_{i=0}^{k} m_{i} x^{i}\right) \cdot\left(\sum_{i=0}^{n-k} n_{i} x^{i}\right)
$$

That is,

$$
P_{m} \equiv\left(\sum_{i=0}^{k} m_{i} x^{i}\right) \cdot\left(\sum_{i=0}^{n-k} n_{i} x^{i}\right)=Q_{m} R_{m}
$$

where $Q_{m}$ and $R_{m}$ are the polynomials $Q, R$, reduced by modulus $m$.

The truth of the theorems is thence immediate.

\section{Illustrative Problems Applying the Tables}

Principles I-III above provide a method for finding the factors of any polynomial by a study of a more limited set of congruent polynomials over finite rings, such as modulo 2,3 , or 5 .

Principle III states that a polynomial is prime, if its homomorphic congruential polynomial in any finite ring (of prime modulus) is prime. It should be remembered that the converse is not true. To determine the reducibility of the following polynomials, we form the corresponding reduced polynomials, with respect to one or more moduli, and note from the Factor Tables whether or not the latter are prime. Thus, to determine reducibility, form $P_{m} \equiv P$ in the following examples:

(a) $x^{3}-11 x^{2}+7 x-16 \equiv x^{3}+x^{2}+x(\bmod 2)$, which is composite, but $P_{3}=x^{3}+x^{2}+x+2(\bmod 3)$, which is prime by the tables; hence $P$ is prime.

(b) $x^{3}-12 x^{2}-17 x+25 \equiv x^{3}+x+1(\bmod 3)$, which is composite. But $P_{2}=x^{3}+x+1(\bmod 2)$, which is prime; hence $P$ is prime.

(c) $x^{4}-13 x^{3}-19 x^{2}+21 x+45 \equiv x^{4}+x^{3}+x^{2}+x+1$ (mod 2) which is prime; hence $P$ is prime. (Although $P_{3}$ and $P_{5}$ are composite).

(d) $x^{4}+7 x^{3}+7 x-35 \equiv x^{4}+x^{3}+x+1(\bmod 2$ and 3$)$.

This is composite in $A_{2}, A_{3}$, and $A_{5}$, so the test is indecisive from the tables alone. It could be tested for factors, as shown below. Actually it is prime by Eisenstein's Theorem.

(e) Given $P=x^{6}-3 x^{5}+5 x^{4}+x^{3}-4 x^{2}+25$. Using the condensed numeration of the tables:

$P_{2}=11111110001=\left(\begin{array}{llll}1 & 7 & 1\end{array}\right)$,

which factors into $(7)(23)=\left(\begin{array}{lll}1 & 1 & 1\end{array}\right)\left(\begin{array}{lllll}1 & 0 & 0 & 1 & 1\end{array}\right)$

$$
=\left(x^{2}+x+1\right)\left(x^{4}+x+1\right) .
$$

Both of these factors are prime, by the tables. It is thus indicated that $P$, if reducible, must factor into a quadratic and a quartic, both prime.

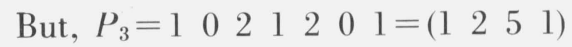

$$
\begin{aligned}
=(47)(54) & =\left(\begin{array}{llll}
1 & 1 & 2 & 1
\end{array}\right)\left(\begin{array}{llll}
1 & 2 & 1 & 1
\end{array}\right) \\
= & \left(x^{3}+x^{2}+2 x+1\right)\left(x^{3}+2 x^{2}+x+1\right) .
\end{aligned}
$$

Both cubics are prime, requiring that $P$ factor, if at all, into two prime cubics. The two factor patterns demanded by $P_{2}$ and $P_{3}$ are incompatible and hence $P$ is irreducible.

(f) Given $\quad P=x^{7}+13 x^{6}-32 x^{5}+27 x^{4}+29 x^{3}+23 x^{2}$

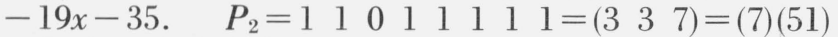
$=\left(\begin{array}{lll}1 & 1 & 1\end{array}\right)\left(\begin{array}{llllll}1 & 0 & 1 & 0 & 0 & 1\end{array}\right)$, a quadratic and a quintic, both prime.

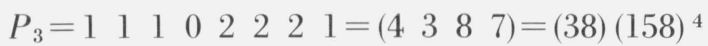

$=\left(\begin{array}{llllll}1 & 0 & 2 & 2\end{array}\right)\left(\begin{array}{llll}1 & 1 & 2 & 2\end{array}\right)$, a cubic and a quartic, both prime.

Since these factor patterns are incompatible, $P$ must be irreducible. It happens here that $P_{5}$ also factors incompatibly into a linear and a sextic, both prime, as the reader may verify. ${ }^{4}$

Further explanations, with both theory and examples will be found in reference [10], but the following will probably prove adequate for a wide variety of factoring problems encountered.

Principles I-III above enable us to find the factors of any polynomial by a study of a more limited set of congruent factors in the finite rings, modulo 2,3 , or 5 . Thus, in modulo 3 , since the prime factors

$$
\left(x^{2}+x+2\right)\left(x^{3}+2 x^{2}+x+1\right)=x^{5}+2 x^{3}+2=P_{3},
$$

then any quintic polynomial $P$ congruent to $P_{3}$, such as

$$
P=x^{5}-3 x^{4}+5 x^{3}-6 x^{2}-36 x-16,
$$

if factorable at all, must have factors congruent to those of $P_{3}$. Actually, here

$$
P=\left(x^{2}-2 x-4\right)\left(x^{3}-x^{2}+7 x+4\right) .
$$

Next will be presented in detail the solution of three typical factoring problems-a quintic, an octic, and a decimic. They are found to be reducible.

EXAMPle I. Quintic

$$
\text { Given } P=x^{5}-6 x^{4}+20 x^{2}+9 x+1 \text {. }
$$

Form the congruential polynomials, moduli 2 and 3 using detached coefficients:

$$
P_{2}=10000011 .
$$

Converting to condensed numeration and entering the tables

$$
P_{2}=\left(\begin{array}{ll}
4 & 3
\end{array}\right)=(7)\left(\begin{array}{ll}
1 & 5
\end{array}\right)=\left(\begin{array}{lll}
1 & 1 & 1
\end{array}\right)\left(\begin{array}{llll}
1 & 1 & 0 & 1
\end{array}\right)
$$

We observe in the tables that both of these factors are prime. This indicates that $P$ factors, if at all, into this pattern-a quadratic times a cubic,-both prime.

Similarly, $P_{3}=1 \quad \begin{array}{llllll}0 & 0 & 2 & 0 & 1=\left(\begin{array}{lll}3 & 2 & 1\end{array}\right)\end{array}$

$$
=\left(\begin{array}{ll}
1 & 5
\end{array}\right)\left(\begin{array}{ll}
5 & 8
\end{array}\right)=\left(\begin{array}{lll}
1 & 1 & 2
\end{array}\right)\left(\begin{array}{llll}
1 & 2 & 2 & 2
\end{array}\right) \text {, both prime. }
$$

${ }_{4}^{4}$ Tables are not available in this article. 
(The cubic (l $\left.\begin{array}{llll}1 & 2 & 2 & 2\end{array}\right)$ is found under its reciprocal (lllll) $\left.\begin{array}{llll}1 & 1 & 1 & 2\end{array}\right)$.

We note here that the factor pattern for $P_{3}$ is consistent with that for $P_{2}$. (If it had been prime, or reducible only as a linear times a quartic, this inconsistency would have necessitated the irreducibility of $P$.)

We now examine the factorization indicated for $P$ :

$$
P=\left(x^{2}+a x+b\right)\left(x^{3}+a^{\prime} x^{2}+b^{\prime} x+c^{\prime}\right) .
$$

Multiplying out, and equating to the known coefficients of $P$ :

$$
\begin{aligned}
a+a^{\prime} & =-6 \\
a a^{\prime}+b+b^{\prime} & =0 \\
a b^{\prime}+a^{\prime} b+c^{\prime} & =20 \\
b b^{\prime}+a c^{\prime} & =9 \\
b c^{\prime} & =1
\end{aligned}
$$

Since the unknown literal coefficients must agree with the known coefficients of the factors of $P_{2}$ and $P_{3}$, we form the table of residues:

\begin{tabular}{r|r|r|r|r|r}
\hline \hline & $a$ & $b$ & $a^{\prime}$ & $b^{\prime}$ & $c^{\prime}$ \\
\cline { 2 - 5 } & 1 & 1 & 1 & 0 & 1 \\
$P_{2} \ldots \ldots \ldots \ldots \ldots$ & 1 & 2 & 2 & 2 & 2 \\
\hline
\end{tabular}

From equation (6), and the residue table, we have $\left(b, c^{\prime}\right)=(-1,-1)$. Then equations $(2)-(5)$ solved simultaneously produce the cubic $a^{3}+6 a^{2}+2 a-15$ $=0$, which promptly yields $a=-5$, with the help of the residue table. Thence $a^{\prime} \equiv-1$ and $b^{\prime}=-4$. So

$$
P=\left(x^{2}-5 x-1\right)\left(x^{3}-x^{2}-4 x-1\right)
$$

in prime factors.

EXAmPle II. Octic

$P=x^{8}+x^{7}-11 x^{6}-2 x^{5}+36 x^{4}-6 x^{3}-43 x^{2}+21 x+24$.

Construct therefrom the reduced congruential functions, for moduli 2, 3, and 5. Using detached coefficients,

$$
\begin{aligned}
& P_{2}=1111100001110 \\
& =\left(\begin{array}{llllllllll}
1 & 0
\end{array}\right)\left(\begin{array}{llllllll}
1 & 1 & 1 & 0 & 0 & 0 & 1 & 1
\end{array}\right)
\end{aligned}
$$

we shall enter the Factor Tables to determine factors of the septic factor

$$
F_{2}=11111000011 .
$$

The reciprocal polynomial must be used here:

$F_{2}^{\prime}=1 \quad 1 \quad 0 \quad 000111$

$=307$, in condensed octic numeration.

$=\left(\begin{array}{ll}2 & 3\end{array}\right)(15)$, prime factors

$=\left(\begin{array}{llllll}1 & 0 & 0 & 1 & 1\end{array}\right)\left(\begin{array}{llll}1 & 1 & 0 & 1\end{array}\right)$

$\therefore F_{2}=\left(\begin{array}{lllll}1 & 1 & 0 & 0 & 1\end{array}\right) \quad\left(\begin{array}{llll}1 & 0 & 1 & 1\end{array}\right)$, quartic $x$ cubic, both prime.

$\therefore P_{2}=\left(\begin{array}{lll}1 & 0\end{array}\right)\left(\begin{array}{lllll}1 & 1 & 0 & 0 & 1\end{array}\right)\left(\begin{array}{llll}1 & 0 & 1 & 1\end{array}\right)$ in prime factors

Next: $P_{3}=1 \quad 1 \quad 1 \quad 1 \quad 0 \quad 0 \quad 2 \quad 0 \quad 0$

$=\left(\begin{array}{lll}1 & 0 & 0\end{array}\right)\left(\begin{array}{lllllll}1 & 1 & 1 & 1 & 0 & 0 & 2\end{array}\right)$.

We shall examine the sextic for factors, using the reciprocal

$$
\begin{aligned}
& F_{3}^{\prime}=10002022 \\
& =1088 \\
& =(5)\left(\begin{array}{lll}
4 & 3 & 7
\end{array}\right) \\
& =(5)(5)\left(\begin{array}{lll}
1 & 6 & 2
\end{array}\right)-\text { all prime factors } \\
& =\left(\begin{array}{lll}
1 & 2
\end{array}\right)\left(\begin{array}{llllll}
1 & 2
\end{array}\right)\left(\begin{array}{lllll}
1 & 2 & 0 & 0 & 2
\end{array}\right) .
\end{aligned}
$$

Again reciprocating,

$$
F_{3}=\left(\begin{array}{lll}
1 & 2
\end{array}\right)\left(\begin{array}{llllll}
1 & 2
\end{array}\right)\left(\begin{array}{lllll}
1 & 0 & 0 & 1 & 2
\end{array}\right)
$$

$\therefore P_{3}=\left(\begin{array}{lll}1 & 0 & 0\end{array}\right)\left(\begin{array}{ll}1 & 2\end{array}\right)\left(\begin{array}{llllll}1 & 2\end{array}\right)\left(\begin{array}{lllll}1 & 0 & 0 & 1 & 2\end{array}\right)$, in prime factors.

Next, $P_{5}=1 \quad 1 \quad 4 \quad 3 \quad 1442114$

$=\left(\begin{array}{llllllllll}1 & 0 & 1 & 2 & 3\end{array}\right)\left(\begin{array}{lllll}1 & 1 & 3 & 0 & 3\end{array}\right)$, both quartics prime.

Use should be made here of the modulus 5 tables, degree 8 (available as mentioned on page 1 above). Without the use of them the problem could be solved, but would involve testing out numerous alternative possibilities, uneconomical in time and effort. The factor pattern of $P_{5}$ signifies that $P$ will factor into 2 prime quartics unless it is prime. Hence let

$$
P=\left(x^{4}+a x^{3}+b x^{2}+c x+d\right)\left(x^{4}+a^{\prime} x^{3}+b^{\prime} x^{2}+c^{\prime} x+d^{\prime}\right) .
$$

Multiplying out and equating to the known coefficients of $P$ :

$$
\begin{aligned}
a+a^{\prime} & =1 \\
a a^{\prime}+b+b^{\prime} & =-11 \\
a b^{\prime}+a^{\prime} b+c+c^{\prime} & =-2 \\
a c^{\prime}+a^{\prime} c+b b^{\prime}+d+d^{\prime} & =36 \\
a d^{\prime}+a^{\prime} d+b c^{\prime}+b^{\prime} c & =-6 \\
b d^{\prime}+b^{\prime} d+c c^{\prime} & =-43 \\
c d^{\prime}+c^{\prime} d & =21 \\
d d^{\prime} & =24
\end{aligned}
$$


Grouping the factors of $P_{2}$ and $P_{3}$ accordingly into (quartic) $x$ (quartic):

$$
\begin{aligned}
& P_{2}=\left(\begin{array}{lllll}
1 & 0 & 1 & 1 & 0
\end{array}\right)\left(\begin{array}{lllll}
1 & 1 & 0 & 0 & 1
\end{array}\right) \\
& P_{3 a}=\left(\begin{array}{llllll}
1 & 1 & 1 & 0 & 0
\end{array}\right)\left(\begin{array}{llllll}
1 & 0 & 0 & 1 & 2
\end{array}\right) \\
& P_{3 b}=\left(\begin{array}{lllll}
1 & 0 & 0 & 1 & 2
\end{array}\right)\left(\begin{array}{llllll}
1 & 1 & 1 & 0 & 0
\end{array}\right) \\
& P_{5 a}=\left(\begin{array}{llllllll}
1 & 0 & 1 & 2 & 3
\end{array}\right)\left(\begin{array}{llllll}
1 & 1 & 3 & 0 & 3
\end{array}\right) \\
& P_{5 b}=\left(\begin{array}{lllll}
1 & 1 & 3 & 0 & 3
\end{array}\right)\left(\begin{array}{lllll}
1 & 0 & 1 & 2 & 3
\end{array}\right)
\end{aligned}
$$

It is observed that $P_{3}$ and $P_{5}$ have two possible ways to relate to $P_{2}$; hence (11) to (14).

The unknown coefficients in (1) must satisfy the following table of residues:

\begin{tabular}{l|c|c|c|c|c|c|c|c}
\hline \hline & $a$ & $b$ & $c$ & $d$ & $a^{\prime}$ & $b^{\prime}$ & $c^{\prime}$ & $d^{\prime}$ \\
\hline$P_{2}$ & 0 & 1 & 1 & 0 & 1 & 0 & 0 & 1 \\
$P_{3 a}$ & 1 & 1 & 0 & 0 & 0 & 0 & 1 & 2 \\
$P_{3 b}$ & 0 & 0 & 1 & 2 & 1 & 1 & 0 & 0 \\
$P_{5 a}$ & 0 & 1 & 2 & 3 & 1 & 3 & 0 & 3 \\
$P_{5 b}$ & 1 & 3 & 0 & 3 & 0 & 1 & 2 & 3 \\
\hline
\end{tabular}

From (9), $d d^{\prime}=24$; then referring to the table of residues, necessarily $\left(d, d^{\prime}\right)=(8,3)$. Thus, $P_{3 a}$ is rejected. Thence, similarly, from $(8),\left(c, c^{\prime}\right)=(7,0)$. Hence $P_{5 b}$ is rejected. $\therefore P_{2}, P_{3 b}$, and $P_{5 a}$ will be used to determine the remaining coefficients.

From $(7),\left(b, b^{\prime}\right)=(-9,-2)$.

Next, from (2) and (3), $\left(a, a^{\prime}\right)=(0,1)$.

The values check in (4), (5), and (6).

$\therefore P=\left(x^{4}-9 x^{2}+7 x+8\right)\left(x^{4}+x^{3}-2 x^{2}+3\right)$, the product of two prime quartics.

Example III. Decimic

$P=x^{10}+19 x^{9}+78 x^{8}-89 x^{7}+15 x^{6}+106 x^{5}$

$$
-45 x^{4}-8 x^{3}+23 x^{2}+15 x+5 .
$$

First, form

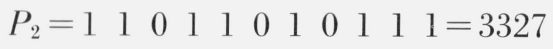

Using the Factor Tables, Degree 10, Mod 2: ${ }^{5}$

$$
P_{2}=(3)\left(\begin{array}{llll}
1 & 1 & 1 & 5
\end{array}\right)=\text { Linear } \times \text { Nonic }
$$$$
=(3)(37)(67)=\text { Linear } \times \text { Quartic } \times \text { Quintic }-
$$
all prime.

$$
\begin{gathered}
=(x+1)\left(x^{4}+x^{3}+x^{2}+x+1\right) \\
\left(x^{5}+x^{4}+x^{2}+x+1\right) .
\end{gathered}
$$

\footnotetext{
${ }^{5}$ Table for this degree not included in this article.
}

Next, $P_{3}=1 \quad 1 \quad 0 \quad 110010112001$.

In this case we must use its reciprocal in the table.

$$
\begin{aligned}
& \therefore P_{3}^{\prime}=100120020016068 \\
& =\left(\begin{array}{lllll}
5
\end{array}\right)\left(\begin{array}{lllll}
4 & 7 & 3 & 2 & 7
\end{array}\right)=\left(\begin{array}{lll}
5
\end{array}\right)\left(\begin{array}{lllll}
5 & 1
\end{array}\right)\left(\begin{array}{llll}
1 & 7 & 3 & 7
\end{array}\right) \\
& =\left(\begin{array}{lll}
1 & 2
\end{array}\right)\left(\begin{array}{llll}
1 & 2 & 0 & 1
\end{array}\right)\left(\begin{array}{lllllll}
1 & 2 & 1 & 1 & 0 & 2 & 1
\end{array}\right) \text {. }
\end{aligned}
$$

We must again reciprocate to examine the cubic and sextic of the tables.

$\therefore\left(P_{3}^{\prime}\right)^{\prime}=P_{3}=\left(\begin{array}{lllllllllllll}1 & 2\end{array}\right)\left(\begin{array}{llllll}1 & 0 & 2 & 1\end{array}\right)\left(\begin{array}{lllllll}1 & 2 & 0 & 1 & 1 & 2 & 1\end{array}\right)$

$=\left(\begin{array}{ll}5\end{array}\right)\left(\begin{array}{llll}3 & 7\end{array}\right)\left(\begin{array}{llll}1 & 6 & 4 & 7\end{array}\right)$. These factors are found to be prime in the tables

$\therefore P_{3}=(x+2)\left(x^{3}+2 x+1\right)\left(x^{6}+2 x^{5}+x^{3}+x^{2}+2 x+1\right)$

$$
=\text { Linear } \times \text { Cubic } \times \text { Sextic, }- \text { all prime. }
$$

Comparing $P_{2}$ and $P_{3}$ we deduce that the only possible factor pattern for $P$ is Sextic $\times$ Quartic, - both prime. (Since, by synthetic division, $P$ has no linear factor.)

Next form

$$
\begin{aligned}
& P_{5}=14431101020300 \\
& =1 \quad 000\left(\begin{array}{lllllllll}
1 & 4 & 3 & 1 & 0 & 1 & 0 & 2 & 3
\end{array}\right) .
\end{aligned}
$$

Examining the octic factor, in the tables for

$$
F_{5}=1443110101028 .
$$

Reciprocating,

$$
\begin{aligned}
& F_{5}^{\prime}=144002002132 \\
& =\left(\begin{array}{llllllllll}
1 & 3
\end{array}\right)\left(\begin{array}{lllllll}
1 & 1 & 2 & 1 & 2 & 1 & 3
\end{array}\right) \\
& =\left(\begin{array}{lll}
1 & 3
\end{array}\right)\left(\begin{array}{llllllll}
1 & 4
\end{array}\right)\left(\begin{array}{lllllll}
1 & 2 & 4 & 0 & 2 & 3 & 1
\end{array}\right) \\
& =\left(\begin{array}{lll}
1 & 3
\end{array}\right)\left(\begin{array}{lllll}
1 & 4
\end{array}\right)\left(\begin{array}{lllll}
1 & 1 & 0 & 1
\end{array}\right)\left(\begin{array}{llll}
1 & 1 & 3 & 1
\end{array}\right) \\
& \therefore F_{5}=\left(\begin{array}{ll}
1 & 2
\end{array}\right)\left(\begin{array}{lll}
1 & 4
\end{array}\right)\left(\begin{array}{llll}
1 & 0 & 1 & 1
\end{array}\right)\left(\begin{array}{llll}
1 & 3 & 1 & 1
\end{array}\right) \text {, all prime } \\
& \therefore P_{5}=1000\left(\begin{array}{lll}
1 & 2
\end{array}\right)\left(\begin{array}{lllll}
1 & 4
\end{array}\right)\left(\begin{array}{llllll}
1 & 0 & 1 & 1
\end{array}\right)\left(\begin{array}{lllll}
1 & 3 & 1 & 1
\end{array}\right) \text {. }
\end{aligned}
$$


Combining the factors of $P_{2}, P_{3}$, and $P_{5}$ into a sextic $\times$ quartic pattern:

$$
\begin{aligned}
& P_{2}=\left(\begin{array}{lllllll}
1 & 0 & 1 & 1 & 0 & 0 & 1
\end{array}\right)\left(\begin{array}{lllll}
1 & 1 & 1 & 1 & 1
\end{array}\right) \\
& P_{3}=\left(\begin{array}{lllllll}
1 & 2 & 0 & 1 & 1 & 2 & 1
\end{array}\right)\left(\begin{array}{llllll}
1 & 2 & 2 & 2 & 2
\end{array}\right) \\
& P_{5 a}=\left(\begin{array}{llllllll}
1 & 0 & 2 & 3 & 2 & 0 & 0
\end{array}\right)\left(\begin{array}{lllll}
1 & 4 & 1 & 0 & 4
\end{array}\right) \\
& P_{5 b}=\left(\begin{array}{llllllllll}
1 & 2 & 3 & 0 & 4 & 0 & 0
\end{array}\right)\left(\begin{array}{llllll}
1 & 2 & 1 & 3 & 2
\end{array}\right) \\
& P_{5 c}=\left(\begin{array}{lllllll}
1 & 4 & 2 & 1 & 4 & 3 & 0
\end{array}\right)\left(\begin{array}{lllll}
1 & 0 & 1 & 1 & 0
\end{array}\right) \\
& P_{5 d}=\left(\begin{array}{lllllll}
1 & 2 & 1 & 3 & 2 & 0 & 0
\end{array}\right)\left(\begin{array}{lllll}
1 & 2 & 3 & 0 & 4
\end{array}\right) \\
& P_{5 e}=\left(\begin{array}{llllllll}
1 & 4 & 1 & 0 & 4 & 0 & 0
\end{array}\right)\left(\begin{array}{llllll}
1 & 0 & 2 & 3 & 2
\end{array}\right) \\
& P_{5 f}=\left(\begin{array}{lllllll}
1 & 1 & 4 & 2 & 4 & 3 & 0
\end{array}\right)\left(\begin{array}{lllll}
1 & 3 & 1 & 1 & 0
\end{array}\right) \\
& P_{5 g}=\left(\begin{array}{lllllll}
1 & 3 & 2 & 0 & 4 & 2 & 1
\end{array}\right)\left(\begin{array}{lllll}
1 & 1 & 3 & 0 & 0
\end{array}\right) .
\end{aligned}
$$

\begin{tabular}{|c|c|c|c|c|c|c|c|c|c|c|}
\hline & $a$ & $b$ & $c$ & $d$ & $e$ & $f$ & $a^{\prime}$ & $b^{\prime}$ & $c^{\prime}$. & $d^{\prime}$ \\
\hline$P_{2} \ldots$ & 0 & 1 & 1 & 0 & 0 & 1 & 1 & 1 & 1 & 1 \\
\hline$P_{3} \ldots$ & 2 & 0 & 1 & 1 & 2 & 1 & 2 & 2 & 2 & 2 \\
\hline .............. & 0 & 2 & 3 & 2 & 0 & 0 & 4 & 1 & 0 & 4 \\
\hline …ㄴ, & 2 & 3 & 0 & 4 & 0 & 0 & 2 & 1 & 3 & 2 \\
\hline$P_{5 c \ldots}$ & 4 & 2 & 1 & 4 & 3 & 0 & 0 & 1 & 1 & 0 \\
\hline$P_{5 d} \ldots$ & 2 & 1 & 3 & 2 & 0 & 0 & 2 & 3 & 0 & 4 \\
\hline$P_{5 e} \ldots$ & 4 & 1 & 0 & 4 & 0 & 0 & 0 & 2 & 3 & 2 \\
\hline$P_{5 f \ldots} \ldots$ & 1 & 4 & 2 & 4 & 3 & 0 & 3 & 1 & 1 & 0 \\
\hline$P_{5 g} \ldots$ & 3 & 2 & 0 & 4 & 2 & 1 & 1 & 3 & 0 & 0 \\
\hline
\end{tabular}

And $P=\left(x^{6}+a x^{5}+b x^{4}+c x^{3}+d x^{2}+e x+f\right)$

$$
\times\left(x^{4}+a^{\prime} x^{3}+b^{\prime} x^{2}+c^{\prime} x+d^{\prime}\right)
$$

The unknown coefficients of $P$ must satisfy the following table of residues (using (1)-(9)):

Multiplying out in (10) and equating to the known coefficients of $P$ :

$$
\begin{aligned}
a+a^{\prime} & =19 \\
a a^{\prime}+b+b^{\prime} & =78 \\
a b^{\prime}+a^{\prime} b+c+c^{\prime} & =-89 \\
a c^{\prime}+a^{\prime} c+b b^{\prime}+d+d^{\prime} & =15 \\
a d^{\prime}+a^{\prime} d+b c^{\prime}+b^{\prime} c+e & =106 \\
a^{\prime} e+b d^{\prime}+b^{\prime} d+c c^{\prime}+f & =-45 \\
a^{\prime} f+b^{\prime} e+c d^{\prime}+c^{\prime} d & =-8 \\
b^{\prime} f+c^{\prime} e+d d^{\prime} & =23
\end{aligned}
$$

$$
\begin{aligned}
c^{\prime} f+d^{\prime} e & =15 \\
d^{\prime} f & =5
\end{aligned}
$$

Using the residue table and eq $(20)$, we have $\left(d^{\prime}, f\right)$ $=(5,1)$, or $\left(d^{\prime}, f\right)=(-1,-5),-$ two possibilities. The first uses $P_{5 g}$, rejecting the other $P_{5}$ 's. The second uses $P_{5 a}$ or $P_{5 d}$ only. Trying the first solution, then from eq (19) we find $\left(c^{\prime}, e\right)=(5,2)$. Thence (18) yields $\left(b^{\prime}, d\right)=(-7,4)$. Next $(17)$ yields $\left(a^{\prime}, c\right)=(11,-5)$. Finally (11) gives $a=8$, and (12) gives $b=-3$. All of these values check in the remaining equations.

$$
\begin{array}{r}
\therefore P=\left(x^{6}+8 x^{5}-3 x^{4}-5 x^{3}+4 x^{2}+2 x+1\right) \\
\left(x^{4}+11 x^{3}-7 x^{2}+5 x+5\right) .
\end{array}
$$

Instead, if we had pursued the wrong possibility $\left(d^{\prime}, f\right)=(-1,-5)$, we would soon encounter unduly large coefficient values and ultimately reach an outright contradiction. For instance, (19) would give $\left(c^{\prime}, e\right)=(5,-40)$; then $(18)$ two sets of values: $\left(b^{\prime}, d\right)$ $=(-37,-38)$ based on $P_{5 d}$, and $\left(b^{\prime}, d\right)=(-49,22)$ based on $P_{5 a}$. Then in (17) the values become outlandishly large, and ennui overcomes valor ere the final kill is attained. One should suspect such wrong choices early and thus avoid lost motion.

\section{Other Long-Range Values of the Factor Tables}

Further values of the factor tables for the pure mathematician might include a study of factor patterns and coefficient sequences. The density and distribution of irreducible polynomials should also be of interest. Much work has been done in this area for the rational integers, but relatively little for polynomials.

In constructing and analyzing Galois Fields, it is necessary in the decomposition of the defining polynomial to know the manner in which polynomials are reducible. The order of the elements of the field and the determination of primitive elements follows therefrom. Expressing the factors merely in terms of their degree is adequate for some purposes, but the exhibition of the actual factors throws additional light on the structure of the field. The understanding of Galois Fields is thus considerably enhanced by this approach.

A recent instance of the application of congruential polynomials is suggested in the work of W. Wesley Peterson on "Error-Correcting Codes" [15]. He deals with error-detecting and error-correcting codes which possess an algebraic structure. He uses the $\mathrm{GF}(2)$ irreducible polynomials in his theory and implementation. He stresses mainly binary codes, since nearly all present-day machines are binary, though there is no purely theoretical basis for this restriction. Undoubtedly the work can and will be carried still further in the future. 
FACTOR TABLES

\begin{tabular}{|c|c|c|c|c|c|c|c|c|c|c|c|}
\hline \multicolumn{12}{|c|}{ Polynomials, Modulo 2} \\
\hline Deg & Poly & Fact & Quot & Deg & Poly & Fact & Quot & Deg & Poly & Fact & Quot \\
\hline 2 & 5 & 3 & 3 & 7 & 225 & 3 & 163 & 8 & 463 & 13 & \\
\hline 2 & 7 & & & 7 & 227 & 7 & 61 & 8 & 465 & 7 & $\begin{array}{l}31 \\
147\end{array}$ \\
\hline 3 & 11 & 3 & 7 & 7 & 231 & 3 & 167 & 8 & 467 & 3 & 355 \\
\hline 3 & 13 & & & 7 & 233 & & 37 & 8 & 471 & & \\
\hline 3 & 17 & 3 & 5 & 7 & 235 & & & 8 & 473 & 3 & 351 \\
\hline 4 & 21 & 3 & 17 & 7 & 237 & 3 & 165 & 8 & 475 & 3 & 353 \\
\hline 4 & 23 & & & 7 & 243 & 3 & 141 & 8 & 477 & & \\
\hline 4 & 25 & 7 & 7 & 7 & 245 & 3 & 143 & 8 & 503 & 3 & 301 \\
\hline 4 & 27 & 3 & 15 & 7 & 247 & & & 8 & 505 & 3 & 303 \\
\hline 4 & 33 & 3 & 11 & 7 & 253 & & & 8 & 507 & 13 & 45 \\
\hline 4 & 37 & & & 7 & 255 & 13 & 23 & 8 & 513 & 7 & 165 \\
\hline 5 & 41 & 3 & 37 & 7 & 257 & 3 & 145 & 8 & 515 & & \\
\hline 5 & 43 & 7 & 15 & 7 & 263 & 7 & 75 & 8 & 517 & 3 & 305 \\
\hline 5 & 45 & & & 7 & 267 & 3 & 155 & 8 & 523 & 15 & 67 \\
\hline 5 & 47 & 3 & 35 & 7 & 273 & 3 & 151 & 8 & 525 & 37 & 37 \\
\hline 5 & 53 & 3 & 31 & 7 & 275 & 3 & 153 & 8 & 527 & 3 & 315 \\
\hline 5 & 55 & 3 & 33 & 7 & 277 & & & 8 & 533 & 3 & 311 \\
\hline 5 & 57 & & & 7 & 303 & 3 & 101 & 8 & 535 & 3 & 313 \\
\hline 5 & 63 & 3 & 21 & 7 & 307 & 15 & 23 & 8 & 537 & & \\
\hline 5 & 67 & & & 7 & 313 & & & 8 & 543 & & \\
\hline 5 & 77 & 3 & 25 & 7 & 317 & 3 & 105 & 8 & 547 & 3 & 335 \\
\hline 6 & 101 & 3 & 77 & 7 & 327 & 3 & 115 & 8 & 553 & 3 & 331 \\
\hline 6 & 103 & & & 7 & 333 & 3 & 111 & 8 & 555 & 3 & 333 \\
\hline 6 & 105 & 13 & 13 & 7 & 337 & 7 & 51 & 8 & 557 & 7 & 171 \\
\hline 6 & 107 & 3 & 75 & 7 & 347 & 3 & 135 & 8 & 563 & 3 & 321 \\
\hline 6 & 111 & & & 7 & 357 & & & 8 & 567 & & \\
\hline 6 & 113 & 3 & 71 & 7 & 377 & 3 & 125 & 8 & 573 & & \\
\hline 6 & 115 & 3 & 73 & 8 & 401 & 3 & 377 & 8 & 575 & 7 & 177 \\
\hline 6 & 117 & 7 & 31 & 8 & 403 & 7 & 155 & 8 & 577 & 3 & 325 \\
\hline 6 & 123 & 3 & 61 & 8 & 405 & 23 & 23 & 8 & 603 & 3 & 201 \\
\hline 6 & 125 & 3 & 63 & 8 & 407 & 3 & 375 & 8 & 607 & & \\
\hline 6 & 127 & & & 8 & 411 & 13 & 57 & 8 & 613 & & \\
\hline 6 & 133 & $\ldots \ldots$ & & 8 & 413 & 3 & 371 & 8 & 617 & 3 & 205 \\
\hline 6 & 135 & 7 & 37 & 8 & 415 & 3 & 373 & 8 & 623 & 7 & 135 \\
\hline 6 & & 3 & 65 & 8 & 417 & 15 & 73 & 8 & 627 & 3 & 215 \\
\hline 6 & 143 & 3 & 41 & 8 & 421 & 7 & $\begin{array}{l}153 \\
\end{array}$ & 8 & 633 & 3 & 211 \\
\hline 6 & 147 & & & 8 & 423 & 3 & 361 & 8 & 637 & & \\
\hline 6 & 153 & 7 & 25 & 8 & 425 & 3 & 363 & 8 & 647 & 3 & 235 \\
\hline 6 & 157 & 3 & 45 & 8 & 427 & 31 & 37 & 8 & 653 & 3 & 231 \\
\hline 6 & 167 & 3 & 55 & 8 & 431 & 3 & 367 & 8 & 657 & 13 & 75 \\
\hline 6 & 177 & 13 & 15 & 8 & 433 & & & 8 & 667 & 7 & 121 \\
\hline 7 & 201 & 3 & 177 & 8 & 435 & $\ldots$ & & 8 & 673 & 23 & 31 \\
\hline 7 & 203 & & & 8 & 437 & 3 & 365 & 8 & 677 & 3 & 225 \\
\hline 7 & 205 & 7 & 67 & 8 & 443 & 3 & 341 & 8 & 707 & 3 & 275 \\
\hline 7 & 207 & 3 & 175 & 8 & 445 & 3 & 343 & 8 & 717 & & \\
\hline 7 & 211 & & & 8 & 447 & 7 & 141 & 8 & 727 & & \\
\hline 7 & 213 & 3 & 171 & 8 & 451 & 3 & 347 & 8 & 737 & 3 & 265 \\
\hline 7 & 215 & 3 & 173 & 8 & 453 & & & 8 & 757 & 3 & 245 \\
\hline 7 & 217 & & & 8 & 455 & & & 8 & 777 & 7 & 111 \\
\hline 7 & 223 & 3 & 161 & 8 & 457 & 3 & 345 & & & & \\
\hline
\end{tabular}


FACTOR TABLES

\begin{tabular}{|c|c|c|c|c|c|c|c|c|c|c|c|}
\hline \multicolumn{12}{|c|}{ Polynomials, Modulo 3} \\
\hline Deg & Poly & Fact & Quot & Deg & Poly & Fact & Quot & Deg & Poly & Fact & Quot \\
\hline 2 & 11 & & & 5 & 301 & 4 & 177 & 5 & 407. & & \\
\hline 2 & 12 & 4 & 5 & 5 & 302 & 5 & 144 & 5 & 408 & 4 & 102 \\
\hline 2 & 14 & 5 & 5 & 5 & 304 & 5 & 145 & 5 & 414. & & \\
\hline 2 & 15 & & & 5 & 305 & 4 & 178 & 5 & 415 & 5 & 181 \\
\hline 2 & 17 & 4 & 4 & 5 & 307 & & & 5 & 417 & 4 & 104 \\
\hline 3 & 31 & 4 & 17 & 5 & 308 & & & 5 & 418. & & \\
\hline 3 & 32 & 5 & 14 & 5 & 311 & 5 & 148 & 5 & 424 & 5 & 185 \\
\hline 3 & 34 & 5 & 15 & 5 & 312 & 18 & 47 & 5 & 425 & 4 & 108 \\
\hline 3 & 35 & 4 & 18 & 5 & 314 & 4 & 171 & 5 & 427 & 11 & 47 \\
\hline 3 & 37 & .... & & 5 & 315 & 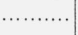 & & 5 & 428 & 18 & 51 \\
\hline 3 & 38 & & & 5 & 317 & 11 & 37 & 5 & 435 & 4 & 118 \\
\hline 3 & 44 & 4 & 11 & 5 & 318 & 4 & 172 & 5 & 437 & 5 & 162 \\
\hline 3 & 45 & $\ldots$. & & 5 & 321 & 15 & 58 & 5 & 444 & 4 & 111 \\
\hline 3 & 47 & $\ldots$ & & 5 & 322 & 4 & 175 & 5 & 445 & 18 & 54 \\
\hline 3 & 48 & 4 & 12 & 5 & 324 & & & 5 & 447 & 10 & T \\
\hline 3 & 55 & 5 & 11 & 5 & 325 & 5 & 141 & 5 & 448 & 4 & 112 \\
\hline 3 & 57 & 4 & 14 & 5 & 327 & 4 & 174 & 5 & 454 & & \\
\hline 4 & 101 & 15 & 18 & 5 & 328 & 11 & 38 & 5 & 455 & & \\
\hline 4 & 102 & 4 & 55 & 5 & 334 & ..... & & 5 & 457 & 4 & 114 \\
\hline 4 & 104 & 5 & 45 & 5 & 335 & $\cdots$ & & 5 & 458 & 5 & 167 \\
\hline 4 & 105 & & & 5 & 337 & 4 & 184 & 5 & 465 & 11 & 45 \\
\hline 4 & 107 & 4 & 54 & 5 & 338 & 5 & 157 & 5 & 467 & 4 & 124 \\
\hline 4 & 108 . & $\ldots$ & & 5 & 341 & 4 & 187 & 5 & 475 & 4 & 128 \\
\hline 4 & 111 & 4 & 57 & 5 & 342 & & & 5 & 477 & 18 & 58 \\
\hline 4 & 112 . & & & 5 & 344 & 18 & 42 & 5 & 478 & 5 & 177 \\
\hline 4 & 114. & & & 5 & 345 & 4 & 188 & 5 & 484 & 4 & 121 \\
\hline 4 & 115 & 4 & 58 & 5 & 347 & 5 & 152 & 5 & 485 & 5 & 171 \\
\hline 4 & 117. & & & 5 & 348 & .... & & 5 & 487 & 5 & 172 \\
\hline 4 & 118 & 5 & 47 & 5 & 351 & & & 5 & 505 & 5 & 101 \\
\hline 4 & 121 & 11 & 11 & 5 & 352 & 5 & 154 & 5 & 507 & 4 & 154 \\
\hline 4 & 124 & 4 & 51 & 5 & 354 & 4 & 181 & 5 & 515 & 4 & 158 \\
\hline 4 & 125 & 5 & 41 & 5 & 355 & 15 & 51 & 5 & 517 & 15 & 42 \\
\hline 4 & 127 & 5 & 42 & 5 & 357 & & & 5 & 525 & 正 & \\
\hline 4 & 128 & 4 & 52 & 5 & 358 & 4 & 182 & 5 & 527 & $\ldots$ & \\
\hline 4 & 134 & 4 & 31 & 5 & 364 & 4 & 161 & 5 & 545 & & \\
\hline 4 & 135 & 11 & 15 & 5 & 365 & 5 & 131 & 5 & 547 & 4 & 134 \\
\hline 4 & 137 & & & 5 & 367 & 5 & 132 & 5 & 555 & 4 & 138 \\
\hline 4 & 138 & 4 & 32 & 5 & 368 & 4 & 162 & 5 & 557 & 5 & 112 \\
\hline 4 & 144. & & & 5 & 372 & 4 & 165 & 5 & 575 & 5 & 121 \\
\hline 4 & 145 & 5 & 51 & 5 & 374 & 5 & 135 & 5 & 587 & 4 & 144 \\
\hline 4 & 147 & 4 & 34 & 5 & 375 & & & 6 & 1001 & 11 & 121 \\
\hline 4 & 148. & & & 5 & 377 & 4 & 164 & 6 & 1002 & 4 & 555 \\
\hline 4 & 154 & 5 & 55 & 5 & 378 & & & 6 & 1004 & 5 & 445 \\
\hline 4 & 155 & 4 & 38 & 5 & 381 & 4 & 167 & 6 & 1005 & & \\
\hline 4 & 157 & 18 & 18 & 5 & 384 & & & 6 & 1007 & 4 & 554 \\
\hline 4 & 165 & 4 & 48 & 5 & 385 & 4 & 168 & 6 & 1008 & & \\
\hline 4 & 167 & 5 & 32 & 5 & 387 & & & 6 & 1011 & 4 & 557 \\
\hline 4 & 175. & & & 5 & 388 & 5 & 137 & 6 & 1012 & 11 & 122 \\
\hline 4 & 177. & $\ldots$ & & 5 & 404 & 4 & 101 & 6 & 1014 & & \\
\hline 4 & 187 & 4 & 44 & 5 & 405 & & & 6 & 1015 & 4 & 558 \\
\hline
\end{tabular}


Factor Tables

\begin{tabular}{|c|c|c|c|c|c|c|c|c|c|c|c|}
\hline \multicolumn{12}{|c|}{ Polynomials, Modulo 3 -Continued } \\
\hline Deg & Poly & Fact & Quot & Deg & Poly & Fact & Quot & Deg & Poly & Fact & Quot \\
\hline 6 & 1017 & & & 6 & 1115 & 5 & 481 & 6 & 1224 & 15 & 175 \\
\hline 6 & 1018 & 5 & 447 & 6 & 1117 & 5 & 482 & 6 & 1225 & 4 & 508 \\
\hline 6 & 1021 & & & 6 & 1118 & 4 & 572 & 6 & 1227 & 18 & 148 \\
\hline 6 & 1022 & 47 & 58 & 6 & 1121 & & & 6 & 1228 & 5 & 407 \\
\hline 6 & 1024 & 4 & 551 & 6 & 1122 & 4 & 575 & 6 & 1234 & 5 & 415 \\
\hline 6 & 1025 & 5 & 441 & 6 & 1124 & 5 & 485 & 6 & 1235 & 4 & 518 \\
\hline 6 & 1027 & 5 & 442 & 6 & 1125 & & & 6 & 1237 & & \\
\hline 6 & 1028 & 4 & 552 & 6 & 1127 & 4 & 574 & 6 & 1238 & 18 & 141 \\
\hline 6 & 1031 & 5 & 458 & 6 & 1128 & & & 6 & 1242 & & \\
\hline 6 & 1032 & 15 & 184 & 6 & 1134 & $\ldots$. & & 6 & 1244 & 4 & 511 \\
\hline 6 & 1034 & 4 & 531 & 6 & 1135 & 5 & 461 & 6 & 1245 & & \\
\hline 6 & 1035 & $\ldots$ & & 6 & 1137 & 4 & 584 & 6 & 1247 & & \\
\hline 6 & 1037 & $\ldots$ & & 6 & 1138 & & & 6 & 1248 & 4 & 512 \\
\hline 6 & 1038 & 4 & 532 & 6 & 1141 & 4 & 587 & 6 & 1251 & 47 & 54 \\
\hline 6 & 1041 & $\ldots$ & & 6 & 1142 & 5 & 464 & 6 & 1254 & 11 & 114 \\
\hline 6 & 1042 & 4 & 535 & 6 & 1144 & 5 & 465 & 6 & 1255 & 5 & 411 \\
\hline 6 & 1044 & 15 & 185 & 6 & 1145 & 4 & 588 & 6 & 1257 & 4 & 514 \\
\hline 6 & 1045 & 5 & 451 & 6 & 1147 & 38 & 38 & 6 & 1258 & 15 & 177 \\
\hline 6 & 1047 & 4 & 534 & 6 & 1148 & & & 6 & 1264 & & \\
\hline 6 & 1048 & $\ldots$ & & 6 & 1151 & 5 & 468 & 6 & 1265 & 15 & 171 \\
\hline 6 & 1051 & 4 & 537 & 6 & 1152 & 18 & 137 & 6 & 1267 & 4 & 524 \\
\hline 6 & 1052 & 5 & 454 & 6 & 1154 & 4 & 581 & 6 & 1268 & 5 & 427 \\
\hline 6 & 1054 & 5 & 455 & 6 & 1155 & 11 & 105 & 6 & 1274 & & \\
\hline 6 & 1055 & 4 & 538 & 6 & 1157 & 15 & 162 & 6 & 1275 & 4 & 528 \\
\hline 6 & 1057 & . & & 6 & 1158 & 4 & 582 & 6 & 1277 & 5 & 422 \\
\hline 6 & 1058 & 42 & 54 & 6 & 1164 & 4 & 561 & 6 & 1278 & & \\
\hline 6 & 1061 & 4 & 547 & 6 & 1165 & & & 6 & 1281 & 45 & 58 \\
\hline 6 & 1064 & .... & & 6 & 1167 & & & 6 & 1284 & 4 & 521 \\
\hline 6 & 1065 & 4 & 548 & 6 & 1168 & 4 & 562 & 6 & 1285 & 18 & 144 \\
\hline 6 & 1067 & 5 & 432 & 6 & 1171 & 5 & 478 & 6 & 1287 & 11 & 117 \\
\hline 6 & 1068 & & & 6 & 1172 & 4 & 565 & 6 & 1288 & 4 & 522 \\
\hline 6 & 1071 & & & 6 & 1174 & 37 & 37 & 6 & 1304 & 4 & 301 \\
\hline 6 & 1072 & 5 & 434 & 6 & 1175 & & & 6 & 1305 & & \\
\hline 6 & 1074 & 4 & 541 & 6 & 1177 & 4 & 564 & 6 & 1307 & 11 & 157 \\
\hline 6 & 1075 & ........ & & 6 & 1178 & 5 & 477 & 6 & 1308 & 4 & 302 \\
\hline 6 & 1077 & 18 & 158 & 6 & 1181 & 4 & 567 & 6 & 1314 & & \\
\hline 6 & 1078 & 4 & 542 & 6 & 1184 & 18 & 132 & 6 & 1315 & $\frac{1}{5}$ & 581 \\
\hline 6 & 1081 & 5 & 438 & 6 & 1185 & 4 & 568 & 6 & 1317 & 4 & 304 \\
\hline 6 & 1082 & 4 & 545 & 6 & 1187 & 5 & 472 & 6 & 1318 & 11 & 158 \\
\hline 6 & 1084 & . & & 6 & 1188 & 11 & 108 & 6 & 1324 & 5 & 585 \\
\hline 6 & 1085 & 45 & 51 & 6 & 1204 & 4 & 501 & 6 & 1325 & 4 & 308 \\
\hline 6 & 1087 & 4 & 544 & 6 & 1205 & 5 & 401 & 6 & 1327 & 54 & 54 \\
\hline 6 & 1088 & 5 & 437 & 6 & 1207 & 5 & 402 & 6 & 1328 & 15 & 117 \\
\hline 6 & 1104 & 42 & 58 & 6 & 1208 & 4 & 502 & 6 & 1334 & & \\
\hline 6 & 1105 & 4 & 578 & 6 & 1212 & 4 & 505 & 6 & 1335 & 4 & 318 \\
\hline 6 & 1107 & 47 & 51 & 6 & 1214 & 5 & 405 & 6 & 1337 & 5 & 562 \\
\hline 6 & 1108 & 5 & 487 & 6 & 1215 & 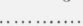 & & 6 & 1338 & 51 & 58 \\
\hline 6 & 1111 & 11 & 101 & 6 & 1217 & 4 & 504 & 6 & 1344 & 4 & 311 \\
\hline 6 & 1112 & 37 & 38 & 6 & 1218 & & & 6 & 1345 & & \\
\hline 6 & 1114 & 4 & 571 & 6 & 1221 & 4 & 507 & 6 & 1347 & 15 & 112 \\
\hline
\end{tabular}


Factor TABles

\begin{tabular}{|c|c|c|c|c|c|c|c|c|c|c|c|}
\hline \multicolumn{12}{|c|}{ Polynomials, Modulo 3-Continued } \\
\hline Deg & Poly & Fact & Quot & Deg & Poly & Fact & Quot & Deg & Poly & Fact & Quot \\
\hline 6 & 1348 & 4 & 312 & 6 & 1524 & 38 & 42 & 6 & 1887 & 4 & 444 \\
\hline 6 & 1354 & 18 & 162 & 6 & 1525 & 5 & 541 & 9 & 47015 & 5 & 17881 \\
\hline 6 & 1355 & & & 6 & 1527 & 4 & 374 & 9 & 47017 & 5 & 17882 \\
\hline 6 & 1357 & 4 & 314 & 6 & 1535 & & & 9 & 47025 & 11 & 4365 \\
\hline 6 & 1358 & 5 & 567 & 6 & 1537 & 4 & 384 & 9 & 47027 & 4 & 12774 \\
\hline 6 & 1364 & 5 & 575 & 6 & 1545 & 4 & 388 & 9 & 47035 & 5 & 17861 \\
\hline 6 & 1365 & 18 & 164 & 6 & 1547 & 5 & 552 & 9 & 47037 & 4 & 12784 \\
\hline 6 & 1367 & 4 & 324 & 6 & 1548 & . & & 9 & 47045 & 4 & 12788 \\
\hline 6 & 1374 . & & & 6 & 1554 & 4 & 381 & 9 & 47047 & 11 & 4367 \\
\hline 6 & 1375 & 4 & 328 & 6 & 1555 & & & 9 & 47048 & 37 & 1355 \\
\hline 6 & 1377 & & & 6 & 1557 & & & 9 & 47054 & 4 & 12781 \\
\hline 6 & 1378 & 5 & 577 & 6 & 1565 & 5 & 531 & 9 & 47055 & 141 & 335 \\
\hline 6 & 1384 & 4 & 321 & 6 & 1567 & 5 & 532 & 9 & 47057 & 164 & 544 \\
\hline 6 & 1385 & 5 & 571 & 6 & 1575 & 37 & 42 & 9 & 47065 & 51 & 1755 \\
\hline 6 & 1387 & 5 & 572 & 6 & 1577 & 4 & 364 & 9 & 47067 & & \\
\hline 6 & 1388 & 4 & 322 & 6 & 1584 & 11 & 144 & 9 & 47075 & 132 & 387 \\
\hline 6 & 1405 & 5 & 501 & 6 & 1585 & 4 & 368 & 9 & 47077 & 4 & 12764 \\
\hline 6 & 1407 & 4 & 354 & 6 & 1587 & & & 9 & 47078 & 5 & 17877 \\
\hline 6 & 1414 & 5 & 505 & 6 & 1605 & 4 & 478 & 9 & 47084 & 18 & 5832 \\
\hline 6 & 1415 & 4 & 358 & 6 & 1607 & 5 & 302 & 9 & 47085 & 4 & 12768 \\
\hline 6 & 1417 & 11 & 137 & 6 & 1615 & 11 & 185 & 9 & 47087 & 5 & 17872 \\
\hline 6 & 1418 & & & 6 & 1617 & & & 9 & 47105 & 5 & 17801 \\
\hline 6 & 1424 & 4 & 351 & 6 & 1625 & 18 & 114 & 9 & 47107 & 5 & 17802 \\
\hline 6 & 1425 & 54 & 58 & 6 & 1627 & 4 & 474 & 9 & 47115 & 38 & 1314 \\
\hline 6 & 1427 & & & 6 & 1635 & 38 & 54 & 9 & 47117 & 4 & 12704 \\
\hline 6 & 1428 & 4 & 352 & 6 & 1637 & 4 & 484 & 9 & 47125 & 4 & 12708 \\
\hline 6 & 1435 & & & 6 & 1645 & 4 & 488 & 9 & 47127 & 18 & 5848 \\
\hline 6 & 1437 & ․․……… & & 6 & 1647 & & & 9 & 47135 & 4 & 12718 \\
\hline 6 & 1444 & ............. & & 6 & 1655 & 5 & 311 & 9 & 47137 & & \\
\hline 6 & 1445 & ............ & & 6 & 1657 & 5 & 312 & 9 & 47145 & 15 & 3161 \\
\hline 6 & 1447 & 4 & 334 & 6 & 1667 & & & 9 & 47147 & & \\
\hline 6 & 1448 & 5 & 517 & 6 & 1675 & 5 & 321 & 9 & 47148 & 4 & 12712 \\
\hline 6 & 1454 & 37 & 47 & 6 & 1677 & 4 & 464 & 9 & 47154 & & \\
\hline 6 & 1455 & 4 & 338 & 6 & 1685 & 4 & 468 & 9 & 47155 & 5 & 17811 \\
\hline 6 & 1457 & 5 & 512 & 6 & 1687 & 15 & 132 & 9 & 47157 & 4 & 12714 \\
\hline 6 & 1458 & & & 6 & 1715 & & & 9 & 47165 & 58 & 1814 \\
\hline 6 & 1465 & 4 & 348 & 6 & 1717 & 4 & 404 & 9 & 47167 & 4 & 12724 \\
\hline 6 & 1467 & 15 & 122 & 6 & 1725 & 4 & 408 & 9 & 47175 & 4 & 12728 \\
\hline 6 & 1474 & 4 & 341 & 6 & 1727 & 5 & 342 & 9 & 47177 & 5 & 17822 \\
\hline 6 & 1475 & 5 & 521 & 6 & 1745 & 5 & 351 & 9 & 47178 & & \\
\hline 6 & 1477 & 5 & 522 & 6 & 1747 & 5 & 352 & 9 & 47184 & 4 & 12721 \\
\hline 6 & 1478 & 4 & 342 & 6 & 1755 & & & 9 & 47185 & 18 & 5844 \\
\hline 6 & 1484 & 5 & 525 & 6 & 1757 & 4 & 414 & 9 & 47187 & 148 & 348 \\
\hline 6 & 1485 & 11 & 135 & 6 & 1775 & 4 & 428 & 9 & 47205 & & \\
\hline 6 & 1487 & 4 & 344 & 6 & 1777 & & & 9 & 47207 & 4 & 12754 \\
\hline 6 & 1505 & 4 & 378 & 6 & 1787 & 38 & 58 & 9 & 47215 & 4 & 12758 \\
\hline 6 & 1507 & 58 & 58 & 6 & 1815 & 4 & 458 & 9 & 47217 & & \\
\hline 6 & 1515 & 15 & 101 & 6 & 1827 & 37 & 51 & 9 & 47225 & 5 & 17841 \\
\hline 6 & 1517 & $\ldots .$. & & 6 & 1845 & & & 9 & 47227 & 5 & 17842 \\
\hline 6 & 1518 & 4 & 372 & 6 & 1857 & 11 & $17 \%$ & 9 & 47235 & 105 & 461 \\
\hline
\end{tabular}


FACTOR TABLES

Polynomials, Modulo 3-Continued

\begin{tabular}{|c|c|c|c|c|c|c|c|c|c|c|c|}
\hline Deg & Poly & Fact & Quot & Deg & Poly & Fact & Quot & Deg & Poly & Fact & Quot \\
\hline 9 & 47237 & 11 & 4387 & 10 & 116567 & 5 & 47532 & 10 & 116647 & 4 & 56234 \\
\hline 9 & 47245 & 5 & 17851 & 10 & 116568 & 371 & 378 & 10 & 116648 & 5 & 47317 \\
\hline 9 & 47247 & 4 & 12734 & 10 & 116571 & & & 10 & 116651 & 4 & 56237 \\
\hline 9 & 47248 & 11 & 4388 & 10 & 116572 & 5 & 47534 & 10 & 116654 & 15 & 16545 \\
\hline 9 & 47254 & 5 & 17855 & 10 & 116574 & 4 & 56141 & 10 & 116655 & 4 & 56238 \\
\hline 9 & 47255 & 4 & 12738 & 10 & 116575 & 177 & 1422 & 10 & 116657 & 5 & 47312 \\
\hline 9 & 47257 & & & 10 & 116577 & 45 & 5342 & 10 & 116658 & & \\
\hline 9 & 47265 & 4 & 12748 & 10 & 116578 & 4 & 56142 & 10 & 116664 & 18 & 13082 \\
\hline 9 & 47267 & 5 & 17832 & 10 & 116581 & 5 & 47538 & 10 & 116665 & 4 & 56248 \\
\hline 9 & 47275 & 171 & 525 & 10 & 116584 & 15 & 16515 & 10 & 116667 & 38 & 3678 \\
\hline 9 & 47277 & 15 & 3172 & 10 & 116585 & 38 & 3664 & 10 & 116668 & 5 & 47327 \\
\hline 9 & 47278 & 4 & 12742 & 10 & 116587 & 4 & 56144 & 10 & 116671 & & \\
\hline 9 & 47284 & & & 10 & 116588 & 5 & 47537 & 10 & 116672 & & \\
\hline 9 & 47285 & & & 10 & 116604 & 148 & 1722 & 10 & 116674 & 4 & 56241 \\
\hline 9 & 47287 & 4 & 12744 & 10 & 116605 & 5 & 47301 & 10 & 116675 & 5 & 47321 \\
\hline 9 & 47305 & 5 & 17601 & 10 & 116607 & 4 & 56254 & 10 & 116677 & 5 & 47322 \\
\hline 9 & 47307 & 4 & 12854 & 10 & 116608 & 37 & 3775 & 10 & 116678 & 4 & 56242 \\
\hline 9 & 47315 & 4 & 12858 & 10 & 116612 & 5 & 47304 & 10 & 116681 & 132 & 1812 \\
\hline 9 & 47317 & 42 & 1215 & 10 & 116614 & 5 & 47305 & 10 & 116684 & 5 & 47325 \\
\hline 9 & 47325 & & & 10 & 116615 & 4 & 56258 & 10 & 116685 & 45 & 5351 \\
\hline 9 & 47327 & 51 & 1737 & 10 & 116617 & 15 & 16542 & 10 & 116687 & 4 & 56244 \\
\hline 9 & 47335 & 15 & 3111 & 10 & 116618 & & & 10 & 116688 & 11 & 10608 \\
\hline 9 & 47337 & 171 & 527 & 10 & 116621 & 5 & 47308 & 10 & 116704 & 5 & 47345 \\
\hline 9 & 47345 & 185 & 571 & 10 & 116624 & 4 & 56251 & 10 & 116705 & 4 & 56278 \\
\hline 9 & 47347 & 4 & 12834 & 10 & 116625 & & & 10 & 116707 & 18 & 13068 \\
\hline 9 & 47348 & 5 & 17617 & 10 & 116627 & & & 10 & 116708 & 54 & 4482 \\
\hline 10 & 116551 & 4 & 56137 & 10 & 116628 & 4 & 56252 & 10 & 116712 & & \\
\hline 10 & 116552 & 5 & 47554 & 10 & 116634 & 4 & 56231 & 10 & 116714 & 4 & 56271 \\
\hline 10 & 116554 & 5 & 47555 & 10 & 116635 & & & 10 & 116715 & & \\
\hline 10 & 116555 & 4 & 56138 & 10 & 116637 & & & 10 & 116717 & 158 & 1678 \\
\hline 10 & 116557 & 185 & 1382 & 10 & 116638 & 4 & 56232 & 10 & 116718 & 4 & 56272 \\
\hline 10 & 116558 & 42 & 5754 & 10 & 116642 & 4 & 56235 & 10 & 116721 & 11 & 10611 \\
\hline 10 & 116564 & 58 & 4172 & 10 & 116644 & 11 & 10604 & 10 & 116724 & 42 & 5738 \\
\hline 10 & 116565 & 4 & 56148 & 10 & 116645 & 162 & 1557 & & & & \\
\hline
\end{tabular}


FACTOR TABLES

\begin{tabular}{|c|c|c|c|c|c|c|c|c|c|c|c|}
\hline \multicolumn{12}{|c|}{ Polynomials, Modulo 5} \\
\hline Deg & Poly & Fact & Quot & Deg & Poly & Fact & Quot & Deg & Poly & Fact & Quot \\
\hline 2 & 101 & 12 & 13 & 3 & 1213 & & & 4 & 10123 & & \\
\hline 2 & 102 & & & 3 & 1214 & & & 4 & 10124 & 12 & 1302 \\
\hline 2 & 103 & & & 3 & 1221 & 11 & 111 & 4 & 10131 & 11 & 1421 \\
\hline 2 & 104 & 11 & 14 & 3 & 1223 & & & 4 & 10132 & & \\
\hline 2 & 111 & ….......... & & 3 & 1224 & 12 & 102 & 4 & 10133 & & \\
\hline 2 & 112 & & & 3 & 1231 & 12 & 103 & 7 & 10134 & 13 & 1203 \\
\hline 2 & 113 & 12 & 14 & 3 & 1232 & 11 & 112 & 4 & 10141 & & 1200 \\
\hline 2 & 114 & 13 & 13 & 3 & 1234 & 14 & 131 & 4 & 10142 & 11 & 1422 \\
\hline 2 & 121 & 11 & 11 & 3 & 1241 & 13 & 142 & 4 & 10143 & 12 & 1304 \\
\hline 2 & 123 & & & 3 & 1243 & 11 & 113 & 4 & 10144 & 14 & 1121 \\
\hline 2 & 124 & & & 3 & 1312 & & & 4 & 10201 & 12 & 1313 \\
\hline 2 & 131 & 14 & 14 & 3 & 1314 & 11 & 124 & 4 & 10203 & & \\
\hline 2 & 132 & 11 & 12 & 3 & $\begin{array}{l}1314 \\
1323\end{array}$ & & & 4 & 10204 & 123 & 133 \\
\hline 2 & 141 & & & 3 & 1324 & 14 & 141 & 4 & 10211 & 14 & 1134 \\
\hline 3 & 1001 & 11 & 141 & 3 & 1331 & 11 & 121 & 4 & 10212 & 111 & 142 \\
\hline 3 & 1002 & 13 & 124 & 3 & 1332 & 12 & 111 & 4 & 10213 & 11 & 1433 \\
\hline 3 & 1003 & 12 & 134 & 3 & 1341 & & & 4 & 10214 & 13 & 1213 \\
\hline 3 & 1004 & 14 & 111 & 3 & 1414 & 12 & 122 & 4 & 10221 & & \\
\hline 3 & 1011 & & & 3 & 1423 & 14 & 102 & 4 & 10222 & 13 & 1214 \\
\hline 3 & 1012 & 11 & 142 & 3 & 1441 & 11 & 131 & 4 & 10223 & & \\
\hline 3 & 1013 & 14 & 112 & 4 & 10001 & 102 & 103 & 4 & 10224 & 11 & 1434 \\
\hline 3 & 1014 & $\ldots$. & & 4 & 10002 & 102 & & 4 & 10231 & & \\
\hline 3 & 1021 & ...... & & 4 & 10003 & …... & & 4 & 10232 & 12 & 1311 \\
\hline 3 & 1022 & 12 & 131 & 4 & 10004 & 11 & 1414 & 4 & 10233 & & \\
\hline 3 & 1023 & 11 & 143 & 4 & 10011 & 12 & 1343 & 4 & 10234 & 14 & 1131 \\
\hline 3 & 1024 & & & 4 & 10012 & 13 & 1244 & 4 & 10241 & 11 & 14.31 \\
\hline 3 & 1031 & 13 & 122 & 4 & 10013 & 14 & 1112 & 4 & 10242 & 112 & 141 \\
\hline 3 & 1032 & ...... & & 4 & 10014 & & & 4 & 10243 & 13 & 1211 \\
\hline 3 & 1033 & …..... & & 4 & 10021 & 11 & 1411 & 4 & 10244 & 12 & 1312 \\
\hline 3 & 1034 & 11 & 144 & 4 & 10022 & 14 & 1113 & 4 & 10301 & 11 & 1441 \\
\hline 3 & 1041 & 12 & 133 & 4 & 10023 & 12 & 1344 & 4 & 10302 & 12 & 1321 \\
\hline 3 & 1042 & & & 4 & 10024 & & & 4 & 10311 & & \\
\hline 3 & 1043 & & & 4 & 10031 & 14 & 1114 & 4 & 10312 & 11 & 1442 \\
\hline 3 & 1044 & 13 & 123 & 4 & 10032 & 11 & 1412 & 4 & 10313 & & \\
\hline 3 & 1111 & 11 & 101 & 4 & 10033 & 13 & 1241 & 4 & 10314 & 12 & 1322 \\
\hline 3 & 1112 & 14 & 123 & 4 & 10034 & & & 4 & 10321 & 12 & 1323 \\
\hline 3 & 1113 & . & & 4 & 10041 & 13 & 1242 & 4 & 10322 & 123 & 134 \\
\hline 3 & 1114 & & & 4 & 10042 & 12 & 1341 & 4 & 10323 & 11 & 1443 \\
\hline 3 & 1121 & 14 & 124 & 4 & 10043 & 11 & 1413 & 4 & 10324 & 14 & 1141 \\
\hline 3 & 1122 & 11 & 102 & 4 & 10044 & & & 4 & 10331 & 13 & 1222 \\
\hline 3 & 1123 & 12 & 144 & 4 & 10101 & 111 & 141 & 4 & 10332 & 124 & 133 \\
\hline 3 & 1124 & 13 & 133 & 4 & 10102 & & & 4 & 10333 & 12 & 1324 \\
\hline 3 & 1131 & & & 4 & 10103 & i11 & 1423 & 4 & 10334 & 11 & 1444 \\
\hline 3 & 1132 & 13 & 134 & 4 & 10104 & 103 & 103 & 4 & 10341 & & \\
\hline 3 & 1134 & & & 4 & 10111 & & & 4 & 10342 & 14 & 1143 \\
\hline 3 & 1141 & & & 4 & 10112 & 12 & 1301 & 4 & 10343 & & \\
\hline 3 & 1142 & 12 & 141 & 4 & 10113 & 13 & 1201 & 4 & 10344 & 13 & 1223 \\
\hline 3 & 1143 & & & 4 & 10114 & 11 & 1424 & 4 & 10401 & 124 & 134 \\
\hline 3 & 1144 & 11 & 104 & 4 & 10121 & 13 & 1202 & 4 & 10411 & 11 & 1401 \\
\hline 3 & 1212 & 12 & 101 & 4 & 10122 & & & 4 & 10412 & & \\
\hline
\end{tabular}


FACTOR TABLES

\begin{tabular}{|c|c|c|c|c|c|c|c|c|c|c|c|}
\hline \multicolumn{12}{|c|}{ Polynomials, Modulo 5-Continued } \\
\hline Deg & Poly & Fact & Quot & Deg & Poly & Fact & Quot & Deg & Poly & Fact & Quot \\
\hline 4 & 10413 & & & 4 & 11223 & 12 & 1444 & 4 & 12114 & 103 & 123 \\
\hline 4 & 10414 & 14 & 1101 & 4 & 11224 & 13 & 1333 & 4 & 12121 & & \\
\hline 4 & 10421 & & & 4 & 11231 & 11 & 1021 & 4 & 12123 & & \\
\hline 4 & 10422 & 11 & 1402 & 4 & 11232 & 13 & 1334 & 4 & 12124 & 14 & 1341 \\
\hline 4 & 10423 & 14 & 1102 & 4 & 11233 & 14 & 1242 & 4 & 12131 & 47 & $5 \pi$ \\
\hline 4 & 10424 & 13 & 1233 & 4 & 11234 & & & 4 & 12132 & 12 & 1011 \\
\hline 4 & 10431 & & & 4 & 11241 & 134 & 134 & 4 & 12134 & & \\
\hline 4 & 10432 & 13 & 1234 & 4 & 11242 & 11 & 1022 & 4 & 12141 & 13 & 1442 \\
\hline 4 & 10433 & 11 & 1403 & 4 & 11243 & 123 & 141 & 4 & 12142 & 14 & 1343 \\
\hline 4 & 10434 & 12 & 1332 & 4 & 11244 & & & 4 & 12143 & 102 & 124 \\
\hline 4 & 10441 & 12 & 1333 & 4 & 11311 & 123 & 142 & 4 & 12212 & 103 & 124 \\
\hline 4 & 10442 & & & 4 & 11312 & 12 & 1401 & 4 & 12213 & 13 & 1401 \\
\hline 4 & 10443 & & & 4 & 11313 & 11 & 1033 & 4 & 12214 & 12 & 1022 \\
\hline 4 & 10444 & 11 & 1404 & 4 & 11314 & 14 & 1201 & 4 & 12221 & 11 & 1111 \\
\hline 4 & 11011 & 11 & 1001 & 4 & 11321 & & & 4 & 12223 & 14 & 1302 \\
\hline 4 & 11012 & 14 & 1223 & 4 & 11322 & 102 & 111 & 4 & 12224 & & \\
\hline 4 & 11013 & & & 4 & 11323 & 14 & 1202 & 4 & 12231 & 133 & 142 \\
\hline 4 & 11014 & 12 & 1422 & 4 & 11324 & 11 & 1034 & 4 & 12232 & 11 & 1112 \\
\hline 4 & 11021 & 12 & 1423 & 4 & 11331 & 12 & 1403 & 4 & 12234 & 13 & 1403 \\
\hline 4 & 11022 & 11 & 1002 & 4 & 11332 & 14 & 1203 & 4 & 12241 & 14 & 1304 \\
\hline 4 & 11023 & & & 4 & 11334 & 124 & 141 & 4 & 12243 & 11 & 1113 \\
\hline 4 & 11024 & & & 4 & 11341 & 11 & 1031 & 4 & 12312 & & \\
\hline 4 & 11031 & 103 & 112 & 4 & 11342 & & & 4 & 12314 & 11 & 1124 \\
\hline 4 & 11032 & & & 4 & 11343 & 12 & 1404 & 4 & 12321 & 111 & 111 \\
\hline 4 & 11034 & 133 & 133 & 4 & 11411 & & & 4 & 12323 & 134 & 142 \\
\hline 4 & 11041 & . & & 4 & 11412 & 11 & 1042 & 4 & 12324 & & \\
\hline 4 & 11042 & & & 4 & 11413 & 12 & 1414 & 4 & 12331 & 11 & 1121 \\
\hline 4 & 11043 & 13 & 1311 & 4 & 11414 & & & 4 & 12332 & & \\
\hline 4 & 11044 & 11 & 1004 & 4 & 11421 & 13 & 1302 & 4 & 12341 & 12 & 1033 \\
\hline 4 & 11111 & 14 & 1234 & 4 & 11423 & 11 & 1043 & 4 & 12342 & 11 & 1122 \\
\hline 4 & 11112 & 133 & 134 & 4 & 11424 & 102 & 112 & 4 & 12343 & 13 & 1411 \\
\hline 4 & 11113 & ...... & & 4 & 11431 & 14 & 1214 & 4 & 12412 & 14 & 1323 \\
\hline 4 & 11114 & & & 4 & 11432 & 12 & 1411 & 4 & 12414 & & \\
\hline 4 & 11121 & 11 & 1011 & 4 & 11434 & 11 & 1044 & 4 & 12421 & 14 & 1324 \\
\hline 4 & 11122 & 12 & 1431 & 4 & 11441 & & & 4 & 12423 & 12 & 1044 \\
\hline 4 & 11123 & 13 & 1321 & 4 & 11442 & 13 & 1304 & 4 & 12424 & 11 & 1134 \\
\hline 4 & 11124 & & & 4 & 11443 & & & 4 & 12431 & 13 & 1422 \\
\hline 4 & 11131 & 13 & 1322 & 4 & 12012 & 11 & 1142 & 4 & 12432 & 111 & 112 \\
\hline 4 & 11132 & 11 & 1012 & 4 & 12013 & & & 4 & 12441 & 11 & 1131 \\
\hline 4 & 11133 & ....... & & 4 & 12014 & ...... & & 4 & 12443 & & \\
\hline 4 & 11134 & 12 & 1432 & 4 & 12021 & & & 4 & 13012 & & \\
\hline 4 & 11141 & 12 & 1433 & 4 & 12023 & 11 & 1143 & 4 & 13014 & 142 & 142 \\
\hline 4 & 11142 & & & 4 & 12024 & 12 & 1002 & 4 & 13023 & & \\
\hline 4 & 11143 & 11 & 1013 & 4 & 12031 & 12 & 1003 & 4 & 13024 & 11 & 1234 \\
\hline 4 & 11144 & 13 & 1323 & 4 & 12032 & 13 & 1434 & 4 & 13031 & & \\
\hline 4 & 11211 & 12 & 1443 & 4 & 12034 & 11 & 1144 & 4 & 13032 & & \\
\hline 4 & 11212 & & & 4 & 12041 & 102 & 123 & 4 & 13041 & 11 & 1231 \\
\hline 4 & 11213 & ... & & 4 & 12043 & 12 & 1004 & 4 & 13112 & 11 & 1242 \\
\hline 4 & 11214 & 11 & 1024 & 4 & 12112 & 13 & 1444 & 4 & 13114 & 13 & 1013 \\
\hline 4 & 11221 & & & 4 & 12113 & 12 & 1014 & 4 & 13123 & 11 & 1243 \\
\hline
\end{tabular}


FACtOR TABLES

Polynomials, Modulo 5-Continued

\begin{tabular}{|c|c|c|c|c|c|c|c|c|c|c|c|}
\hline Deg & Poly & Fact & Quot & Deg & Poly & Fact & Quot & Deg & Poly & Fact & Quot \\
\hline 4 & 13124 & ... & & 5 & 100033 & 12 & 13424 & 5 & 100311 & 102 & 1033 \\
\hline 4 & 13131 & & & 5 & 100034 & 11 & 14144 & 5 & 100312 & 12 & 13401 \\
\hline 4 & 13132 & 14 & 1403 & 5 & 100041 & $\ldots \ldots$ & & 5 & 100313 & & \\
\hline 4 & 13141 & 14 & 1404 & 5 & 100042 & & & 5 & 100314 & 11 & 14124 \\
\hline 4 & 13143 & 13 & 1011 & 5 & 100043 & -..... & & 5 & 100321 & 123 & 1312 \\
\hline 4 & 13214 & 111 & 124 & 5 & 100044 & & & 5 & 100322 & 13 & 12414 \\
\hline 4 & 13223 & 13 & 1021 & 5 & 100101 & 142 & 1143 & 5 & 100323 & & \\
\hline 4 & 13224 & 12 & 1102 & 5 & 100102 & & & 5 & 100324 & 12 & 13402 \\
\hline 4 & 13231 & 12 & 1103 & 5 & 100103 & 12 & 13434 & 5 & 100331 & 11 & 14121 \\
\hline 4 & 13232 & & & 5 & 100104 & 13 & 12443 & 5 & 100332 & 141 & 1102 \\
\hline 4 & 13241 & & & 5 & 100111 & 11 & 14101 & 5 & 100333 & 14 & 11142 \\
\hline 4 & 13243 & 12 & 1104 & 5 & 100112 & 13 & 12444 & 5 & 100334 & & 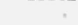 \\
\hline 4 & 13312 & 14 & 1423 & 5 & 100113 & 103 & 1021 & 5 & 100341 & & \\
\hline 4 & 13314 & .... & & 5 & 100114 & & & 5 & 100342 & 11 & 14122 \\
\hline 4 & 13323 & ..... & & 5 & 100121 & 14 & 11124 & 5 & 100343 & 12 & 13404 \\
\hline 4 & 13331 & 141 & 141 & 5 & 100122 & 11 & 14102 & 5 & 100344 & 111 & 1404 \\
\hline 4 & 13332 & 11 & 1212 & 5 & 100123 & 112 & 1444 & 5 & 100401 & 12 & 13413 \\
\hline 4 & 13341 & & & 5 & 100124 & & & 5 & 100402 & 11 & 14132 \\
\hline 4 & 13414 & 11 & 1224 & 5 & 100131 & 124 & 1304 & 5 & 100403 & & \\
\hline 4 & 13423 & & & 5 & 100132 & & & 5 & 100404 & 112 & 1442 \\
\hline 4 & 13431 & 11 & 1221 & 5 & 100133 & 11 & 14103 & 5 & 100411 & & \\
\hline 4 & 13432 & & & 5 & 100134 & 12 & 13432 & 5 & 100412 & 103 & 1024 \\
\hline 4 & 13441 & 13 & 1042 & 5 & 100141 & 12 & 13433 & 5 & 100413 & 11 & 14133 \\
\hline 4 & 14014 & 11 & 1324 & 5 & 100142 & 134 & 1203 & 5 & 100414 & 14 & 11101 \\
\hline 4 & 14023 & 13 & 1121 & 5 & 100143 & & & 5 & 100421 & & \\
\hline 4 & 14041 & 14 & 1004 & 5 & 100144 & 11 & 14104 & 5 & 100422 & 142 & 1141 \\
\hline 4 & 14114 & 12 & 1222 & 5 & 100201 & & & 5 & 100423 & 13 & 12421 \\
\hline 4 & 14123 & & & 5 & 100202 & 14 & 11133 & 5 & 100424 & 11 & 14134 \\
\hline 4 & 14132 & 13 & 1134 & 5 & 100203 & 123 & 1311 & 5 & 100431 & 13 & 12422 \\
\hline 4 & 14141 & 11 & 1331 & 5 & 100204 & 11 & 14114 & 5 & 100432 & 12 & 13411 \\
\hline 4 & 14214 & & & 5 & 100211 & 12 & 13443 & 5 & 100433 & & \\
\hline 4 & 14223 & 11 & 1343 & 5 & 100212 & & & 5 & 100434 & 134 & 1201 \\
\hline 4 & 14241 & 12 & 1233 & 5 & 100213 & 13 & 12401 & 5 & 100441 & 11 & 14131 \\
\hline 4 & 14332 & 102 & 141 & 5 & 100214 & 102 & 1032 & 5 & 100442 & & \\
\hline 4 & 14341 & 112 & 133 & 5 & 100221 & 11 & 14111 & 5 & 100443 & 124 & 1302 \\
\hline 4 & 14441 & & & 5 & 100222 & & & 5 & 100444 & 12 & 13412 \\
\hline 5 & 100001 & 11 & 14141 & 5 & 100223 & 12 & 13444 & 5 & 101011 & 134 & 1214 \\
\hline 5 & 100002 & 12 & 13421 & 5 & 100224 & 133 & 1213 & 5 & 101012 & 12 & 13001 \\
\hline 5 & 100003 & 13 & 12431 & 5 & 100231 & & & 5 & 101013 & 11 & 14233 \\
\hline 5 & 100004 & 14 & 11111 & 5 & 100232 & 11 & 14112 & 5 & 101014 & 124 & 1311 \\
\hline 5 & 100011 & 13 & 12432 & 5 & 100233 & 111 & 1403 & 5 & 101021 & 13 & 12002 \\
\hline 5 & 100012 & 11 & 14142 & 5 & 100234 & 13 & 12403 & 5 & 101022 & & \\
\hline 5 & 100013 & 14 & 11112 & 5 & 100241 & 141 & 1101 & 5 & 101023 & & \\
\hline 5 & 100014 & 12 & 13422 & 5 & 100242 & 12 & 13441 & 5 & 101024 & 11 & 14234 \\
\hline 5 & 100021 & 12 & 13423 & 5 & 100243 & 11 & 14113 & 5 & 101031 & 12 & 13003 \\
\hline 5 & 100022 & 14 & 11113 & 5 & 100244 & & & 5 & 101032 & & \\
\hline 5 & 100023 & 11 & 14143 & 5 & 100301 & 13 & 12412 & 5 & 101033 & ..... & \\
\hline 5 & 100024 & 13 & 12433 & 5 & 100302 & 133 & 1214 & 5 & 101034 & 13 & 12003 \\
\hline 5 & 100031 & 14 & 11114 & 5 & 100303 & 11 & 14123 & 5 & 101041 & 11 & 14231 \\
\hline 5 & 100032 & 13 & 12434 & 5 & 100304 & & & 5 & 101042 & 13 & 12004 \\
\hline
\end{tabular}


Factor TABles

Polynomials, Modulo 5-Continued

\begin{tabular}{|c|c|c|c|c|c|c|c|c|c|c|c|}
\hline Deg & Poly & Fact & Quot & Deg & Poly & Fact & Quot & Deg & Poly & Fact & Quot \\
\hline 5 & 101043 & 12 & 13004 & 5 & 101322 & 12 & 13031 & 5 & 102111 & 12 & 13143 \\
\hline 5 & 101044 & 14 & 11221 & 5 & 101323 & 14 & 11202 & 5 & 102112 & & \\
\hline 5 & 101101 & 11 & 14241 & 5 & 101324 & 13 & 12033 & 5 & 102113 & 11 & 14333 \\
\hline 5 & 101102 & 14 & 11233 & 5 & 101331 & 102 & 1043 & 5 & 102114 & & \\
\hline 5 & 101103 & & & 5 & 101332 & 11 & 14212 & 5 & 102121 & & \\
\hline 5 & 101104 & & & 5 & 101333 & 14.1 & 1113 & 5 & 102122 & & \\
\hline 5 & 101111 & 14 & 11234 & 5 & 101334 & 12 & 13032 & 5 & 102123 & 12 & 13144 \\
\hline 5 & 101112 & 11 & 14242 & 5 & 101341 & 12 & 13033 & 5 & 102124 & 11 & 14334 \\
\hline 5 & 101113 & 12 & 13014 & 5 & 101342 & 142 & 1101 & 5 & 102131 & & \\
\hline 5 & 101114 & 13 & 12013 & 5 & 101343 & 11 & 14213 & 5 & 102132 & 13 & 12134 \\
\hline 5 & 101121 & 111 & 1411 & 5 & 101344 & 103 & 1033 & 5 & 102133 & 14 & 11342 \\
\hline 5 & 101122 & 13 & 12014 & 5 & 101401 & ........ & & 5 & 102134 & & \\
\hline 5 & 101123 & 11 & 14243 & 5 & 101402 & & & 5 & 102141 & 11 & 14331 \\
\hline 5 & 101124 & 123 & 1323 & 5 & 101403 & 11 & 14223 & 5 & 102142 & 12 & 13141 \\
\hline 5 & 101131 & 112 & 1403 & 5 & 101404 & 12 & 13042 & 5 & 102143 & 134 & 1222 \\
\hline 5 & 101132 & 12 & 13011 & 5 & 101411 & 12 & 13043 & 5 & 102144 & 112 & 1412 \\
\hline 5 & 101133 & 124 & 1312 & 5 & 101412 & 13 & 12044 & 5 & 102201 & 11 & 14341 \\
\hline 5 & 101134 & 11 & 14244 & 5 & 101413 & 14 & 11212 & 5 & 102203 & & \\
\hline 5 & 101141 & ..... & & 5 & 101414 & 11 & 14224 & 5 & 102204 & 13 & 12143 \\
\hline 5 & 101142 & $\ldots .$. & & 5 & 101421 & 133 & 1222 & 5 & 102211 & & \\
\hline 5 & 101143 & 13 & 12011 & 5 & 101422 & 14 & 11213 & 5 & 102212 & 11 & 14342 \\
\hline 5 & 101144 & 12 & 13012 & 5 & 101423 & 12 & 13044 & 5 & 102213 & & \\
\hline 5 & 101201 & 14 & 11244 & 5 & 101424 & 141 & 1114 & 5 & 102214 & 14 & 11301 \\
\hline 5 & 101202 & 12 & 13021 & 5 & 101431 & 11 & 14221 & 5 & 102221 & 103 & 1042 \\
\hline 5 & 101203 & & & 5 & 101432 & 134 & 1213 & 5 & 102222 & 134 & 1223 \\
\hline 5 & 101204 & & & 5 & 101433 & 13 & 12041 & 5 & 102223 & 11 & 14343 \\
\hline 5 & 101211 & 11 & 14201 & 5 & 101434 & 142 & 1102 & 5 & 102224 & 12 & 13102 \\
\hline 5 & 101212 & & & 5 & 101441 & 13 & 12042 & 5 & 102231 & 12 & 13103 \\
\hline 5 & 101213 & & & 5 & 101442 & 11 & 14222 & 5 & 102232 & 14 & 11303 \\
\hline 5 & 101214 & 12 & 13022 & 5 & 101443 & & & 5 & 102233 & 13 & 12141 \\
\hline 5 & 101221 & 12 & 13023 & 5 & 101444 & & & 5 & 102234 & 11 & 14344 \\
\hline 5 & 101222 & 11 & 14202 & 5 & 102011 & 14 & 11334 & 5 & 102241 & 13 & 12142 \\
\hline 5 & 101223 & 13 & 12021 & 5 & 102012 & & & 5 & 102242 & & \\
\hline 5 & 101224 & 14 & 11241 & 5 & 102013 & & & 5 & 102243 & 12 & 13104 \\
\hline 5 & 101231 & 13 & 12022 & 5 & 102014 & 11 & 14324 & 5 & 102244 & & \\
\hline 5 & 101232 & 111 & 1412 & 5 & 102021 & & & 5 & 102301 & 12 & 13113 \\
\hline 5 & 101233 & 11 & 14203 & 5 & 102022 & 12 & 13131 & 5 & 102302 & & \\
\hline 5 & 101234 & 102 & 1042 & 5 & 102023 & 13 & 12121 & 5 & 102304 & 14 & 11311 \\
\hline 5 & 101241 & 103 & 1032 & 5 & 102024 & & & 5 & 102311 & 11 & 14301 \\
\hline 5 & 101242 & 14 & 11243 & 5 & 102031 & 11 & 14321 & 5 & 102312 & & \\
\hline 5 & 101243 & 112 & 1404 & 5 & 102032 & 112 & 1411 & 5 & 102313 & 12 & 13114 \\
\hline 5 & 101244 & 11 & 14204 & 5 & 102033 & 142 & 1114 & 5 & 102314 & & \\
\hline 5 & 101301 & ....... & & 5 & 102034 & 12 & 13132 & 5 & 102321 & 13 & 12102 \\
\hline 5 & 101302 & $\begin{array}{l}\cdots . . \\
\ldots . . \\
\end{array}$ & & 5 & 102041 & 12 & 13133 & 5 & 102322 & 11 & 14302 \\
\hline 5 & 101304 & 11 & 14214 & 5 & 102042 & 11 & 14322 & 5 & 102323 & 124 & 1322 \\
\hline 5 & 101311 & 13 & 12032 & 5 & 102043 & 14 & 11332 & 5 & 102324 & 103 & 1043 \\
\hline 5 & 101312 & ..... & & 5 & 102044 & 13 & 12123 & 5 & 102331 & 14 & 11314 \\
\hline 5 & 101313 & ..... & & 5 & 102102 & 11 & 14332 & 5 & 102332 & 12 & 13111 \\
\hline 5 & 101314 & 14 & 11201 & 5 & 102103 & 13 & 12131 & 5 & 102333 & 11 & 14303 \\
\hline 5 & 101321 & 11 & 14211 & 5 & 102104 & 12 & 13142 & 5 & 102334 & 13 & 12103 \\
\hline
\end{tabular}


Factor Tables

\begin{tabular}{|c|c|c|c|c|c|c|c|c|c|c|c|}
\hline \multicolumn{12}{|c|}{ Polynomials, Modulo 5-Continued } \\
\hline Deg & Poly & Fact & Quot & Deg & Poly & Fact & Quot & Deg & Poly & Fact & Quot \\
\hline 5 & 102341 & & & 5 & 103133 & 12 & 13224 & 5 & 103424 & 12 & 13202 \\
\hline 5 & 102342 & 13 & 12104 & 5 & 103134 & 13 & 12203 & 5 & 103431 & 12 & 13203 \\
\hline 5 & 102343 & & & 5 & 103141 & 14 & 11404 & 5 & 103432 & 13 & 12234 \\
\hline 5 & 102344 & 11 & 14304 & 5 & 103142 & 11 & 14422 & 5 & 103433 & 11 & 14403 \\
\hline 5 & 102401 & 13 & 12112 & 5 & 103143 & & & 5 & 103434 & 14 & 11431 \\
\hline 5 & 102403 & 14 & 11322 & 5 & 103144 & & & 5 & 103441 & & \\
\hline 5 & 102411 & & & 5 & 103203 & 12 & 13234 & 5 & 103442 & & \\
\hline 5 & 102412 & 14 & 11323 & 5 & 103204 & 14 & 11411 & 5 & 103443 & 12 & 13204 \\
\hline 5 & 102413 & & & 5 & 103211 & & & 5 & 103444 & 11 & 14404 \\
\hline 5 & 102414 & 12 & 13122 & 5 & 103212 & 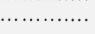 & & 5 & 104011 & 11 & 14001 \\
\hline 5 & 102421 & 11 & 14311 & 5 & 103213 & 11 & 14433 & 5 & 104012 & 111 & 1442 \\
\hline 5 & 102422 & 13 & 12114 & 5 & 103214 & 13 & 12213 & 5 & 104013 & 141 & 1143 \\
\hline 5 & 102423 & & & 5 & 103221 & & & 5 & 104014 & 13 & 12313 \\
\hline 5 & 102424 & $\ldots$ & & 5 & 103222 & 12 & 13231 & 5 & 104021 & & \\
\hline 5 & 102431 & $\cdots$ & & 5 & 103223 & & & 5 & 104022 & 111 & 14002 \\
\hline 5 & 102432 & 11 & 14312 & 5 & 103224 & 11 & 14434 & 5 & 104023 & 12 & 13344 \\
\hline 5 & 102433 & 12 & $13124 H$ & 5 & $10323 \mathrm{I}$ & 14 & 11414 & 5 & 104024 & & \\
\hline 5 & 102434 & & & 5 & 103232 & & & 5 & 104031 & & \\
\hline 5 & 102441 & 142 & 1113 & 5 & 103233 & & & 5. & 104032 & 14 & 11003 \\
\hline 5 & 102442 & 124 & 1323 & 5 & 103234 & 12 & 13232 & 5 & 104033 & 11 & 14003 \\
\hline 5 & 102443 & 11 & 14313 & 5 & 103241 & 11 & 14431 & 5 & 104034 & & \\
\hline 5 & 102444 & 14 & 11321 & 5 & 103242 & 133 & 1244 & 5 & 104041 & 14 & 11004 \\
\hline 5 & 103011 & & & 5 & 103243 & 13 & 12211 & 5 & 104042 & 12 & 13341 \\
\hline 5 & 103012 & 13 & 12244 & 5 & 103244 & 141 & 1134 & 5 & 104043 & 13 & 12311 \\
\hline 5 & 103013 & 12 & 13214 & 5 & 103301 & 11 & 14441 & 5 & 104044 & 11 & 14004 \\
\hline 5 & 103014 & & & 5 & 103302 & 13 & 12224 & 5 & 104104 & 11 & 14014 \\
\hline 5 & 103021 & 11 & 14411 & 5 & 103311 & 12 & 13243 & 5 & 104111 & & \\
\hline 5 & 103022 & & & 5 & 103312 & 11 & 14442 & 5 & 104112 & 12 & 13301 \\
\hline 5 & 103023 & & & 5 & 103313 & & & 5 & 104113 & 14 & 11012 \\
\hline 5 & 103024 & 14 & 1144.1 & 5 & 103314 & & & 5 & 104114 & & \\
\hline 5 & 103031 & 133 & 1242 & 5 & 103321 & 14 & 11424 & 5 & 104121 & 11 & 14011 \\
\hline 5 & 103032 & 11 & 14412 & 5 & 103322 & & & 5 & 104122 & 14 & 11013 \\
\hline 5 & 103033 & 13 & 12241 & 5 & 103323 & 11 & 14443 & 5 & 104123 & 13 & 12321 \\
\hline 5 & 103034 & 123 & 1343 & 5 & 103324 & & & 5 & 104124 & 12 & 13302 \\
\hline 5 & 103041 & 13 & 12242 & 5 & 103331 & 13 & 12222 & 5 & 104131 & 12 & 13303 \\
\hline 5 & 103042 & 14 & 11443 & 5 & 103332 & & & 5 & 104132 & 11 & 14012 \\
\hline 5 & 103043 & 11 & 14413 & 5 & 103333 & & & 5 & 104133 & 103 & 1011 \\
\hline 5 & 103044 & 12 & 13212 & 5 & 103334 & 11 & 14444 & 5 & 104134 & 124 & 1341 \\
\hline 5 & 103102 & 12 & 13221 & 5 & 103341 & 111 & 1431 & 5 & 10414.1 & 123 & 1302 \\
\hline 5 & 103104 & ..... & & 5 & 103342 & 12 & 13241 & 5 & 104142 & 102 & 1021 \\
\hline 5 & 103111 & & & 5 & 103343 & 123 & 1341 & 5 & 104143 & 11 & 14013 \\
\hline 5 & 103112 & & & 5 & 103344 & 13 & 12223 & 5 & 104144 & 13 & 12323 \\
\hline 5 & 103113 & 13 & 12201 & 5 & 103401 & & & 5 & 104203 & 11 & 14023 \\
\hline 5 & 103114 & 11 & 14424 & 5 & 103411 & 11 & 14401 & 5 & 104211 & 13 & 12332 \\
\hline 5 & 103121 & 12 & 13223 & 5 & 103412 & 12 & 13201 & 5 & 104212 & 14 & 11023 \\
\hline 5 & 103122 & 102 & 1011 & 5 & 103413 & …. & & 5 & 104213 & 12 & 13314 \\
\hline 5 & 103123 & 14 & 11402 & 5 & 103414 & & & 5 & 104214 & 11 & 14024 \\
\hline 5 & 103124 & 111 & 1434 & 5 & 103421 & 141 & 1131 & 5 & 104221 & 14 & 11024 \\
\hline 5 & 103131 & 11 & 14421 & 5 & 103422 & 11 & 14402 & 5 & 104222 & 112 & 1431 \\
\hline 5 & 103132 & 14 & 11403 & 5 & 103423 & 102 & 1014 & 5 & 104223 & 134 & 1242 \\
\hline
\end{tabular}


FACTOR TABLES

Polynomials, Modulo 5-Continued

\begin{tabular}{|c|c|c|c|c|c|c|c|c|c|c|c|}
\hline Deg & Poly & Fact & Quot & Deg & Poly & Fact & Quot & Deg & Poly & Fact & Quot \\
\hline 5 & 104224 & 13 & 12333 & 5 & 110031 & 13 & 13122 & 5 & 110334 & 13 & 13103 \\
\hline 5 & 104231 & 11 & 14021 & 5 & 110032 & 12 & 14211 & 5 & 110341 & 11 & 10031 \\
\hline 5 & 104232 & 12 & 13311 & 5 & 110034 & 112 & 1032 & 5 & 110342 & 12 & 14241 \\
\hline 5 & 104233 & 133 & 1201 & 5 & 110041 & & & 5 & 110343 & & \\
\hline 5 & 104234 & 111 & 1444 & 5 & 110042 & 142 & 1201 & 5 & 110344 & 124 & 1431 \\
\hline 5 & 104241 & & & 5 & 110043 & 134 & 1322 & 5 & 110411 & & \\
\hline 5 & 104242 & 11 & 14022 & 5 & 110044 & 11 & 10004 & 5 & 110412 & 11 & 10042 \\
\hline 5 & 104243 & & & 5 & 110111 & 13 & 13132 & 5 & 110413 & 14 & 12212 \\
\hline 5 & 104244 & 12 & 13312 & 5 & 110112 & 111 & 1042 & 5 & 110414 & 13 & 13113 \\
\hline 5 & 104311 & 14 & 11034 & 5 & 110113 & 102 & 1134 & 5 & 110421 & & \\
\hline 5 & 104312 & 13 & 12344 & 5 & 110114 & 12 & 14222 & 5 & 110422 & 13 & 13114 \\
\hline 5 & 104313 & 11 & 14033 & 5 & 110121 & 11 & 10011 & 5 & 110423 & 11 & 10043 \\
\hline 5 & 104314 & 12 & 13322 & 5 & 110122 & 134 & 1323 & 5 & 110424 & 12 & 14202 \\
\hline 5 & 104321 & 12 & 13323 & 5 & 110123 & & & 5 & 110431 & 12 & 14203 \\
\hline 5 & 104322 & 124 & 1343 & 5 & 110124 & 13 & 13133 & 5 & 110432 & & \\
\hline 5 & 104323 & 142 & 1134 & 5 & 110131 & & & 5 & 110433 & 141 & 1213 \\
\hline 5 & 104324 & 11 & 14034 & 5 & 110132 & 11 & 10012 & 5 & 110434 & 11 & 10044 \\
\hline 5 & 104331 & 141 & 1141 & 5 & 110133 & 12 & 14224 & 5 & 110441 & & \\
\hline 5 & 104332 & 123 & 1304 & 5 & 110134 & 14 & 12231 & 5 & 110442 & & \\
\hline 5 & 104333 & 12 & 13324 & 5 & 110141 & 112 & 1033 & 5 & 110443 & 12 & 14204 \\
\hline 5 & 104334 & 14 & 11031 & 5 & 110142 & & & 5 & 110444 & & \\
\hline 5 & 104341 & 11 & 14031 & 5 & 110143 & 11 & 10013 & 5 & 111012 & 11 & 10142 \\
\hline 5 & 104342 & & & 5 & 110144 & & & 5 & 111013 & & \\
\hline 5 & 104343 & 14 & 11032 & 5 & 110211 & 123 & 1442 & 5 & 111014 & 123 & 1403 \\
\hline 5 & 104344 & & & 5 & 110212 & 13 & 13144 & 5 & 111021 & & \\
\hline 5 & 104401 & 11 & 14041 & 5 & 110213 & & & 5 & 111023 & 11 & 10143 \\
\hline 5 & 104411 & & & 5 & 110214 & 11 & 10024 & 5 & 111024 & & \\
\hline 5 & 104412 & 11 & 14042 & 5 & 110221 & 142 & 1203 & 5 & 111031 & 102 & 1143 \\
\hline 5 & 104413 & 13 & 12301 & 5 & 110222 & 12 & 14231 & 5 & 111032 & & \\
\hline 5 & 104414 & 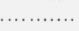 & & 5 & 110223 & 111 & 1043 & 5 & 111034 & 11 & 10144 \\
\hline 5 & 104421 & 13 & 12302 & 5 & 110224 & 14 & 12241 & 5 & 111041 & 13 & 13242 \\
\hline 5 & 104422 & 12 & 13331 & 5 & 110231 & 11 & 10021 & 5 & 111042 & 12 & 14341 \\
\hline 5 & 104423 & 11 & 14043 & 5 & 110232 & & & 5 & 111043 & 14 & 12332 \\
\hline 5 & 104424 & 14 & 11041 & 5 & 110233 & 13 & 13141 & 5 & 111111 & 11 & 10101 \\
\hline 5 & 104431 & 134 & 1244 & 5 & 110234 & 12 & 14232 & 5 & 111112 & 12 & 14301 \\
\hline 5 & 104432 & 103 & 1014 & 5 & 110241 & 12 & 14233 & 5 & 111113 & 13 & 13201 \\
\hline 5 & 104433 & 14 & 11042 & 5 & 110242 & 11 & 10022 & 5 & 111114 & & \\
\hline 5 & 104434 & 11 & 14044 & 5 & 110243 & & & 5 & 111121 & 13 & 13202 \\
\hline 5 & 104441 & 12 & 13333 & 5 & 110244 & & & 5 & 111122 & 11 & 10102 \\
\hline 5 & 104442 & 13 & 12304 & 5 & 110311 & 12 & 14243 & 5 & 111123 & & \\
\hline 5 & 104443 & 102 & 1024 & 5 & 110312 & 102 & 1131 & 5 & 111124 & 12 & 14302 \\
\hline 5 & 104444 & 133 & 1203 & 5 & 110313 & 11 & 10033 & 5 & 111131 & 12 & 14303 \\
\hline 5 & 110011 & 11 & 10001 & 5 & 110314 & 14 & 12201 & 5 & 111132 & 123 & 1404 \\
\hline 5 & 110012 & 14 & 12223 & 5 & 110321 & 13 & 13102 & 5 & 111133 & 11 & 10103 \\
\hline 5 & 110013 & 12 & 14214 & 5 & 110322 & & & 5 & 111134 & 13 & 13203 \\
\hline 5 & 110014 & & & 5 & 110323 & 12 & 14244 & 5 & 111141 & 142 & 1213 \\
\hline 5 & 110021 & 14 & 12224 & 5 & 110324 & 11 & 10034 & 5 & 111142 & 13 & 13204 \\
\hline 5 & 110022 & 11 & 10002 & 5 & 110331 & & & 5 & 111143 & 12 & 14304 \\
\hline 5 & 110023 & 13 & 13121 & 5 & 110332 & 14 & 12203 & 5 & 111144 & 11 & 10104 \\
\hline 5 & 110024 & 141 & 1214 & 5 & 110333 & & & 5 & 111211 & 112 & 1043 \\
\hline
\end{tabular}


FACtor TABLES

\begin{tabular}{|c|c|c|c|c|c|c|c|c|c|c|c|}
\hline \multicolumn{12}{|c|}{ Polynomials, Modulo 5-Continued } \\
\hline Deg & Poly & Fact & Quot & Deg & Poly & Fact & Quot & Deg & Poly & Fact & Quot \\
\hline 5 & 111212 & & & 5 & 112023 & & & 5 & 112342 & & \\
\hline 5 & 111213 & 12 & 14314 & 5 & 112024 & 11 & 10234 & 5 & 112343 & 11 & 10213 \\
\hline 5 & 111214 & 13 & 13213 & 5 & 112031 & 124 & 1404 & 5 & 112344 & 14 & 12421 \\
\hline 5 & 111221 & 11 & 10111 & 5 & 112032 & ........ & & 5 & 112411 & 14 & 12434 \\
\hline 5 & 111222 & 13 & 13214 & 5 & 112034 & & & 5 & 112412 & 124 & 1403 \\
\hline 5 & 111223 & 14 & 12302 & 5 & 112041 & 11 & 10231 & 5 & 112413 & 12 & 14414 \\
\hline 5 & 111224 & & & 5 & 112042 & 14 & 12443 & 5 & 112414 & 11 & 10224 \\
\hline 5 & 111231 & & & 5 & 112043 & 13 & 13311 & 5 & 112421 & 13 & 13302 \\
\hline 5 & 111232 & 111 & 10112 & 5 & 112112 & 11 & 10242 & 5 & 112423 & 103 & 1141 \\
\hline 5 & 111233 & 142 & 1214 & 5 & 112113 & & & 5 & 112424 & 142 & 1222 \\
\hline 5 & 111234 & & & 5 & 112114 & 14 & 12401 & 5 & 112431 & 11 & 10221 \\
\hline 5 & 111241 & 14 & 12304 & 5 & 112121 & 123 & 1412 & 5 & 112432 & 12 & 14411 \\
\hline 5 & 111242 & 103 & 1134 & 5 & 112122 & 12 & 14431 & 5 & 112433 & & \\
\hline 5 & 111243 & 11 & 10113 & 5 & 112123 & 11 & 10243 & 5 & 112434 & 13 & 13303 \\
\hline 5 & 111244 & 12 & 14312 & 5 & 112124 & 103 & 1143 & 5 & 112441 & & \\
\hline 5 & 111311 & . & & 5 & 112131 & 13 & 13322 & 5 & 112442 & 11 & 10222 \\
\hline 5 & 111312 & $\cdots$ & & 5 & 112132 & 14 & 12403 & 5 & 112443 & 14 & 12432 \\
\hline 5 & 111313 & 14 & 12312 & 5 & 112133 & & & 5 & 113012 & 12 & 14001 \\
\hline 5 & 111314 & 11 & 10124 & 5 & 112134 & 11 & 10244 & 5 & 113013 & 112 & 1014 \\
\hline 5 & 111321 & 12 & 14323 & 5 & 112141 & 12 & 14433 & 5 & 113014 & 11 & 10324 \\
\hline 5 & 111322 & 14 & 12313 & 5 & 112142 & & & 5 & 113021 & 102 & 1113 \\
\hline 5 & 111323 & 13 & 13221 & 5 & 112143 & & & 5 & 113023 & 14 & 12002 \\
\hline 5 & 111324 & & & 5 & 112144 & 13 & 13323 & 5 & 113024 & 12 & 14002 \\
\hline 5 & 111331 & 11 & 10121 & 5 & 112211 & 11 & 10201 & 5 & 113031 & 11 & 10321 \\
\hline 5 & 111332 & 102 & 1141 & 5 & 112212 & & & 5 & 113032 & 13 & 13434 \\
\hline 5 & 111334 & & & 5 & 112213 & 14 & 12412 & 5 & 113034 & & \\
\hline 5 & 111341 & 124 & 1444 & 5 & 112214 & & & 5 & 113041 & 14 & 12004 \\
\hline 5 & 111342 & 11 & 10122 & 5 & 112221 & 111 & 1011 & 5 & 113042 & 11 & 10322 \\
\hline 5 & 111343 & 141 & 1223 & 5 & 112223 & 12 & 14444 & 5 & 113043 & 12 & 14004 \\
\hline 5 & 111344 & 13 & 13223 & 5 & 112224 & 13 & 13333 & 5 & 113112 & 13 & 13444 \\
\hline 5 & 111411 & 13 & 13232 & 5 & 112231 & 14 & 12414 & 5 & 113113 & 11 & 10333 \\
\hline 5 & 111412 & 14 & 12323 & 5 & 112232 & 13 & 13334 & 5 & 113114 & 111 & 1024 \\
\hline 5 & 111413 & 11 & 10133 & 5 & 112233 & 11 & 10203 & 5 & 113121 & 134 & 1304 \\
\hline 5 & 111414 & 133 & 1343 & 5 & 112234 & & & 5 & 113122 & 14 & 12013 \\
\hline 5 & 111421 & 14 & 12324 & 5 & 112241 & & & 5 & 113123 & 102 & 1114 \\
\hline 5 & 111422 & 12 & 14331 & 5 & 112242 & 12 & 14441 & 5 & 113124 & 11 & 10334 \\
\hline 5 & 111423 & & & 5 & 112243 & & & 5 & 113131 & 14 & 12014 \\
\hline 5 & 111424 & 11 & 10134 & 5 & 112244 & 11 & 10204 & 5 & 113132 & 12 & 14011 \\
\hline 5 & 111431 & & & 5 & 112311 & & & 5 & 113134 & & \\
\hline 5 & 111432 & 13 & 13234 & 5 & 112312 & 12 & 14401 & 5 & 113141 & 11 & 10331 \\
\hline 5 & 111433 & & & 5 & 112313 & & & 5 & 113142 & & \\
\hline 5 & 111434 & 12 & 14332 & 5 & 112314 & & & 5 & 113143 & ….. & \\
\hline 5 & 111441 & 11 & 10131 & 5 & 112321 & 11 & 10211 & 5 & 113144 & 12 & 14012 \\
\hline 5 & 111442 & & & 5 & 112322 & 133 & 1304 & 5 & 113212 & 11 & 10342 \\
\hline 5 & 111443 & 103 & 1131 & 5 & 112323 & & & 5 & 113213 & 13 & 13401 \\
\hline 5 & 111444 & 14 & 12321 & 5 & 112324 & 12 & 14402 & 5 & 113214 & 12 & 14022 \\
\hline 5 & 112012 & & & 5 & 112331 & 12 & 14403 & 5 & 113221 & 12 & 14023 \\
\hline 5 & 112013 & 11 & 10233 & 5 & 112332 & 11 & 10212 & 5 & 113223 & 11 & 10343 \\
\hline 5 & 112014 & 12 & 14422 & 5 & 112334 & & & 5 & 113224 & & \\
\hline 5 & 112021 & 12 & 14423 & 5 & 112341 & 13 & 13342 & 5 & 113231 & $\cdots$ & \\
\hline
\end{tabular}


FACTOR TABLES

Polynomials, Modulo 5-Continued

\begin{tabular}{|c|c|c|c|c|c|c|c|c|c|c|c|}
\hline Deg & Poly & Fact & Quot & Deg & Poly & Fact & Quot & Deg & Poly & Fact & Quot \\
\hline 5 & 113232 & 112 & 1011 & 5 & 114123 & 12 & 14144 & 5 & 120021 & 11 & 11411 \\
\hline 5 & 113233 & 12 & 14024 & 5 & 114124 & 134 & 1311 & 5 & 120023 & 134 & 1442 \\
\hline 5 & 113234 & 11 & 10344 & 5 & 114131 & 11 & 10421 & 5 & 120024 & 12 & 10002 \\
\hline 5 & 113241 & & & 5 & 114132 & & & 5 & 120031 & 12 & 10003 \\
\hline 5 & 113242 & 13 & 13404 & 5 & 114134 & 103 & 1113 & 5 & 120032 & 11 & 11412 \\
\hline 5 & 113243 & & & 5 & 114141 & & & 5 & 120034 & 14 & 13331 \\
\hline 5 & 113244 & 14 & 12021 & 5 & 114142 & 11 & 10422 & 5 & 120041 & 111 & 1131 \\
\hline 5 & 113311 & 11 & 10301 & 5 & 114143 & 13 & 13011 & 5 & 120043 & 11 & 11413 \\
\hline 5 & 113312 & & & 5 & 114144 & 14 & 12121 & 5 & 120112 & 142 & 1311 \\
\hline 5 & 113313 & 133 & 1311 & 5 & 114212 & 12 & 14101 & 5 & 120113 & 12 & 10014 \\
\hline 5 & 113314 & 13 & 13413 & 5 & 114213 & 11 & 10433 & 5 & 120114 & 11 & 11424 \\
\hline 5 & 113321 & & & 5 & 114214 & 142 & 1242 & 5 & 120121 & 124 & 1014 \\
\hline 5 & 113322 & 11 & 10302 & 5 & 114221 & 133 & 1322 & 5 & 120123 & 13 & 14321 \\
\hline 5 & 113323 & & & 5 & 114223 & 13 & 13021 & 5 & 120124 & 14 & 13341 \\
\hline 5 & 113324 & ...... & & 5 & 114224 & 11 & 10434 & 5 & 120131 & 11 & 11421 \\
\hline 5 & 113331 & 111 & 1021 & 5 & 114231 & 12 & 14103 & 5 & 120132 & 12 & 10011 \\
\hline 5 & 113332 & & & 5 & 114232 & 103 & 1114 & 5 & 120134 & & \\
\hline 5 & 113334 & 12 & 14032 & 5 & 114233 & & & 5 & 120141 & & \\
\hline 5 & 113341 & 12 & 14033 & 5 & 114234 & 14 & 12131 & 5 & 120142 & 11 & 11422 \\
\hline 5 & 113342 & & & 5 & 114241 & 11 & 10431 & 5 & 120143 & & \\
\hline 5 & 113343 & 13 & 13411 & 5 & 114242 & & & 5 & 120212 & & \\
\hline 5 & 113411 & 12 & 14043 & 5 & 114243 & 12 & 14104 & 5 & 120213 & 11 & 11433 \\
\hline 5 & 113412 & & & 5 & 114312 & 11 & 10442 & 5 & 120214 & 12 & 10022 \\
\hline 5 & 113413 & 134 & 1302 & 5 & 114313 & 12 & 14114 & 5 & 120221 & 12 & 10023 \\
\hline 5 & 113414 & 124 & 1411 & 5 & 114314 & & & 5 & 120223 & 14 & 13302 \\
\hline 5 & 113421 & 11 & 10311 & 5 & 114321 & & & 5 & 120224 & 11 & 11434 \\
\hline 5 & 113423 & 12 & 14044 & 5 & 114323 & 11 & 10443 & 5 & 120231 & 134 & 1444 \\
\hline 5 & 113424 & 14 & 12041 & 5 & 114324 & 13 & 13033 & 5 & 120232 & 13 & 14334 \\
\hline 5 & 113431 & 13 & 13422 & 5 & 114331 & & & 5 & 120234 & & \\
\hline 5 & 113432 & 11 & 10312 & 5 & 114332 & 12 & 14111 & 5 & 120241 & 11 & 11431 \\
\hline 5 & 113434 & & & 5 & 114334 & 11 & 10444 & 5 & 120242 & & \\
\hline 5 & 113441 & 133 & 1312 & 5 & 114341 & 141 & 1201 & 5 & 120243 & & \\
\hline 5 & 113442 & 12 & 14041 & 5 & 114342 & 14 & 12143 & 5 & 120312 & 11 & 11442 \\
\hline 5 & 113443 & 11 & 10313 & 5 & 114343 & & & 5 & 120313 & 14 & 13312 \\
\hline 5 & 114012 & & & 5 & 114411 & 11 & 10401 & 5 & 120314 & 124 & 1011 \\
\hline 5 & 114013 & 13 & 13001 & 5 & 114412 & 13 & 13044 & 5 & 120321 & & \\
\hline 5 & 114014 & & & 5 & 114413 & 123 & 1431 & 5 & 120323 & 11 & 11443 \\
\hline 5 & 114021 & 11 & 10411 & 5 & 114414 & 12 & 14122 & 5 & 120324 & 111 & 1134 \\
\hline 5 & 114023 & 141 & 1203 & 5 & 114421 & 12 & 14123 & 5 & 120331 & 14 & 13314 \\
\hline 5 & 114024 & 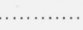 & & 5 & 114423 & 14 & 12102 & 5 & 120332 & & \\
\hline 5 & 114031 & 14 & 12114 & 5 & 114424 & & & 5 & 120334 & 11 & 11444 \\
\hline 5 & 114032 & 11 & 10412 & 5 & 114431 & & & 5 & 120341 & 12 & 10033 \\
\hline 5 & 114034 & 12 & 14132 & 5 & 114432 & 14 & 12103 & 5 & 120342 & 123 & 1024 \\
\hline 5 & 114041 & 12 & 14133 & 5 & 114434 & & & 5 & 120343 & & \\
\hline 5 & 114042 & 13 & 13004 & 5 & 114441 & 13 & 13042 & 5 & 120412 & 14 & 13323 \\
\hline 5 & 114043 & 11 & 10413 & 5 & 114442 & & & 5 & 120413 & 13 & 14301 \\
\hline 5 & 114112 & 14 & 12123 & 5 & 114443 & 142 & 1244 & 5 & 120414 & 103 & 1223 \\
\hline 5 & 114113 & 111 & 1033 & 5 & 120012 & 12 & 10001 & 5 & 120421 & 13 & 14302 \\
\hline 5 & 114114 & 11 & 10424 & 5 & 120013 & & & 5 & 120423 & 12 & 10044 \\
\hline 5 & 114121 & 14 & 12124 & 5 & 120014 & 13 & 14313 & 5 & 120424 & & \\
\hline
\end{tabular}


Factor Tables

\begin{tabular}{|c|c|c|c|c|c|c|c|c|c|c|c|}
\hline \multicolumn{12}{|c|}{ Polynomials, Modulo 5-Continued } \\
\hline Deg & Poly & Fact & Quot & Deg & Poly & Fact & Quot & Deg & Poly & Fact & Quot \\
\hline 5 & 120431 & & & 5 & 121413 & & & 5 & 122342 & 11 & 11122 \\
\hline 5 & 120432 & & & 5 & 121414 & 12 & 10122 & 5 & 122343 & 14 & 13032 \\
\hline 5 & 120434 & 13 & 14303 & 5 & 121421 & 12 & 10123 & 5 & 122412 & 12 & 10201 \\
\hline 5 & 120441 & & & 5 & 121423 & 11 & 11043 & 5 & 122413 & 11 & 11133 \\
\hline 5 & 120442 & 12 & 10041 & 5 & 121424 & & & 5 & 122414 & & \\
\hline 5 & 120443 & 141 & 1323 & 5 & 121431 & 13 & 14422 & 5 & 122421 & & \\
\hline 5 & 121012 & ........ & & 5 & 121432 & 10 & & 5 & 122423 & & \\
\hline 5 & 121014 & & & 5 & 121434 & 11 & 11044 & 5 & 122424 & 11 & 11134 \\
\hline 5 & 121023 & & & 5 & 121441 & & & 5 & 122431 & 12 & 10203 \\
\hline 5 & 121024 & 13 & 14433 & 5 & 121442 & 134 & 1403 & 5 & 122432 & 124 & 1033 \\
\hline 5 & 121031 & & & 5 & 121443 & 14 & 13432 & 5 & 122441 & 11 & 11131 \\
\hline 5 & 121032 & 13 & 14434 & 5 & 122012 & 11 & 11142 & 5 & 122443 & 12 & 10204 \\
\hline 5 & 121041 & 12 & 10133 & 5 & 122014 & 14 & 13001 & 5 & 123012 & 133 & 1434 \\
\hline 5 & 121043 & & & 5 & 122023 & 11 & 11143 & 5 & 123014 & & \\
\hline 5 & 121112 & 13 & 14444 & 5 & 122024 & 134 & 1411 & 5 & 123023 & 12 & 10344 \\
\hline 5 & 121113 & 133 & 1411 & 5 & 122031 & 141 & 1341 & 5 & 123024 & 11 & 11234 \\
\hline 5 & 121114 & 14 & 13401 & 5 & 122032 & 12 & 10211 & 5 & 123031 & 13 & 14122 \\
\hline 5 & 121121 & 11 & 11011 & 5 & 122041 & 14 & 13004 & 5 & 123032 & 141 & 1302 \\
\hline 5 & 121123 & 12 & 10144 & 5 & 122043 & & & 5 & 123041 & 11 & 11231 \\
\hline 5 & 121124 & 142. & 1322 & 5 & 122112 & & & 5 & 123043 & 111 & 1113 \\
\hline 5 & 121131 & & & 5 & 122113 & 14 & 13012 & 5 & 123112 & 11 & 11242 \\
\hline 5 & 121132 & 11 & 11012 & 5 & 122114 & 12 & 10222 & 5 & 123114 & & \\
\hline 5 & 121134 & 102 & 1242 & 5 & 122123 & & & 5 & 123123 & 11 & 11243 \\
\hline 5 & 121141 & 13 & 14442 & 5 & 122124 & & & 5 & 123124 & 12 & 10302 \\
\hline 5 & 121142 & 12 & 10141 & 5 & 122131 & 14 & 13014 & 5 & 123131 & 12 & 10303 \\
\hline 5 & 121143 & 11 & 11013 & 5 & 122132 & & & 5 & 123132 & 13 & 14134 \\
\hline 5 & 121212 & 12 & 10101 & 5 & 122134 & 123 & 1043 & 5 & 123134 & 11 & 11244 \\
\hline 5 & 121213 & 13 & 14401 & 5 & 122141 & ....... & & 5 & 123141 & ...... & \\
\hline 5 & 121214 & 11 & 11024 & 5 & 122142 & & & 5 & 123142 & & \\
\hline 5 & 121221 & 13 & 14402 & 5 & 122143 & 13 & 14011 & 5 & 123143 & 12 & 10304 \\
\hline 5 & 121223 & & & 5 & 122212 & 14 & 13023 & 5 & 123212 & 13 & 14144 \\
\hline 5 & 121224 & 12 & 10102 & 5 & 122213 & 141 & 1343 & 5 & 123213 & 12 & 10314 \\
\hline 5 & 121231 & 11 & 11021 & 5 & 122214 & & & 5 & 123214 & 141 & 1304 \\
\hline 5 & 121232 & & & 5 & 122221 & 11 & 11111 & 5 & 123223 & 133 & 1431 \\
\hline 5 & 121234 & 13 & 14403 & 5 & 122223 & 13 & 14021 & 5 & 123224 & & \\
\hline 5 & 121241 & 133 & 1412 & 5 & 122224 & & & 5 & 123231 & & \\
\hline 5 & 121242 & 11 & 11022 & 5 & 122231 & 13 & 14022 & 5 & 123232 & 12 & 10311 \\
\hline 5 & 121243 & 12 & 10104 & 5 & 122232 & 11 & 11112 & 5 & 123234 & 14 & 13131 \\
\hline 5 & 121312 & 14 & 13423 & 5 & 122234 & 12 & 10232 & 5 & 123241 & 13 & 14142 \\
\hline 5 & 121314 & 13 & 14413 & 5 & 122241 & 12 & 10233 & 5 & 123243 & 14 & 13132 \\
\hline 5 & 121321 & 14 & 13424 & 5 & 122243 & 11 & 11113 & 5 & 123312 & 112 & 1101 \\
\hline 5 & 121323 & 111 & 1143 & 5 & 122312 & & & 5 & 123314 & 12 & 10322 \\
\hline 5 & 121324 & 11 & 11034 & 5 & 122314 & 11 & 11124 & 5 & 123321 & 11 & 11211 \\
\hline 5 & 121331 & 123 & 1032 & 5 & 122321 & 103 & 1242 & 5 & 123323 & 102 & 1214 \\
\hline 5 & 121332 & 12 & 10111 & 5 & 122323 & 12 & 10244 & 5 & 123324 & 14 & 13141 \\
\hline 5 & 121334 & & & 5 & 122324 & 13 & 14033 & 5 & 123331 & & \\
\hline 5 & 121341 & 11 & 11031 & 5 & 122331 & 11 & 11121 & 5 & 123332 & 11 & 11212 \\
\hline 5 & 121342 & & & 5 & 122332 & 13 & 14034 & 5 & 123341 & & \\
\hline 5 & 121343 & 13 & 14411 & 5 & 122334 & 14 & 13031 & 5 & 123342 & 13 & 14104 \\
\hline 5 & 121412 & 11 & 11042 & 5 & 122341 & & & 5 & 123343 & 11 & 11213 \\
\hline
\end{tabular}


Factor TABles

Polynomials, Modulo 5-Continued

\begin{tabular}{|c|c|c|c|c|c|c|c|c|c|c|c|}
\hline Deg & Poly & Fact & Quot & Deg & Poly & Fact & Quot & Deg & Poly & Fact & Quot \\
\hline 5 & 123412 & & & 5 & 124441 & 134 & 1434 & 5 & 131132 & 13 & 10134 \\
\hline 5 & 123414 & 11 & 11224 & 5 & 124443 & 11 & 11313 & 5 & 131141 & 12 & 11433 \\
\hline 5 & 123421 & & & 5 & 130012 & & & 5 & 131143 & 124 & 1102 \\
\hline 5 & 123423 & 14 & 13102 & 5 & 130014 & 11 & 12324 & 5 & 131212 & 13 & 10144 \\
\hline 5 & 123424 & 112 & 1102 & 5 & 130023 & 12 & 11344 & 5 & 131214 & 134 & 1021 \\
\hline 5 & 123431 & 11 & 11221 & 5 & 130024 & 14 & 14441 & 5 & 131223 & 12 & 11444 \\
\hline 5 & 123432 & 14 & 13103 & 5 & 130031 & 11 & 12321 & 5 & 131224 & 11 & 12434 \\
\hline 5 & 123441 & 12 & 10333 & 5 & 130032 & 133 & 1024 & 5 & 131231 & & \\
\hline 5 & 123443 & 13 & 14111 & 5 & 130041 & 123 & 1102 & 5 & 131232 & 102 & 1341 \\
\hline 5 & 124012 & 13 & 14244 & 5 & 130112 & 12 & 11301 & 5 & 131241 & 11 & 12431 \\
\hline 5 & 124014 & 11 & 11324 & 5 & 130114 & 13 & 10013 & 5 & 131243 & & \\
\hline 5 & 124023 & & & 5 & 130123 & 14 & 14402 & 5 & 131312 & 11 & 12442 \\
\hline 5 & 124024 & & & 5 & 130124 & 11 & 12334 & 5 & 131314 & 112 & 1222 \\
\hline 5 & 124031 & 11 & 11321 & 5 & 130131 & 12 & 11303 & 5 & 131323 & 11 & 12443 \\
\hline 5 & 124032 & 103 & 1214 & 5 & 130132 & 14 & 14403 & 5 & 131324 & 12 & 11402 \\
\hline 5 & 124041 & 13 & 14242 & 5 & 130141 & 11 & 12331 & 5 & 131331 & 12 & 11403 \\
\hline 5 & 124043 & & & 5 & 130143 & 12 & 11304 & 5 & 131332 & & \\
\hline 5 & 124112 & 123 & 1014 & 5 & 130212 & 11 & 12342 & 5 & 131341 & & \\
\hline 5 & 124114 & & & 5 & 130214 & 103 & 1323 & 5 & 131412 & 123 & 1114 \\
\hline 5 & 124123 & & & 5 & 130223 & 11 & 12343 & 5 & 131414 & 13 & 10113 \\
\hline 5 & 124124 & 11 & 11334 & 5 & 130224 & & & 5 & 131423 & 142 & 1434 \\
\hline 5 & 124131 & 133 & 1442 & 5 & 130231 & 13 & 10022 & 5 & 131424 & 14 & 14041 \\
\hline 5 & 124132 & & & 5 & 130232 & 12 & 11311 & 5 & 131431 & 102 & 1343 \\
\hline 5 & 124134 & 12 & 10432 & 5 & 130241 & & & 5 & 131432 & 12 & 11411 \\
\hline 5 & 124141 & 11 & 11331 & 5 & 130243 & 133 & 1021 & 5 & 131441 & & \\
\hline 5 & 124143 & 14 & 13232 & 5 & 130312 & 14 & 14423 & 5 & 131443 & 13 & 10111 \\
\hline 5 & 124212 & 11 & 11342 & 5 & 130314 & 12 & 11322 & 5 & 132014 & 133 & 1043 \\
\hline 5 & 124213 & 112 & 1114 & 5 & 130323 & & & 5 & 132023 & 103 & 1341 \\
\hline 5 & 124214 & 13 & 14213 & 5 & 130324 & 13 & 10033 & 5 & 132032 & & \\
\hline 5 & 124223 & 11 & 11343 & 5 & 130331 & & & 5 & 132041 & 13 & 10242 \\
\hline 5 & 124224 & 14 & 13241 & 5 & 130332 & 13 & 10034 & 5 & 132112 & 14 & 14123 \\
\hline 5 & 124231 & & & 5 & 130341 & & & 5 & 132114 & 142 & 1442 \\
\hline 5 & 124232 & & & 5 & 130343 & & & 5 & 132123 & & \\
\hline 5 & 124241 & 102 & 1223 & 5 & 130412 & 13 & 10044 & 5 & 132124 & & \\
\hline 5 & 124243 & 13 & 14211 & 5 & 130414 & & & 5 & 132132 & 11 & 12012 \\
\hline 5 & 124312 & 12 & 10401 & 5 & 130423 & 123 & 1101 & 5 & 132141 & & \\
\hline 5 & 124314 & 14 & 13201 & 5 & 130424 & 134 & 1011 & 5 & 132143 & 11 & 12013 \\
\hline 5 & 124323 & 13 & 14221 & 5 & 130431 & & & 5 & 132214 & 11 & 12024 \\
\hline 5 & 124324 & 12 & 10402 & 5 & 130432 & 11 & 12312 & 5 & 132223 & 141 & 1403 \\
\hline 5 & 124331 & 12 & 10403 & 5 & 130441 & 12 & 11333 & 5 & 132224 & 103 & 1343 \\
\hline 5 & 124332 & 14 & 13203 & 5 & 130443 & 11 & 12313 & 5 & 132231 & 11 & 12021 \\
\hline 5 & 124341 & 14 & 13204 & 5 & 131014 & 12 & 11422 & 5 & 132232 & & \\
\hline 5 & 124343 & 12 & 10404 & 5 & 131023 & 13 & 10121 & 5 & 132241 & & \\
\hline 5 & 124412 & & & 5 & 131032 & 11 & 12412 & 5 & 132243 & 13 & 10211 \\
\hline 5 & 124414 & $\cdots \cdots$ & & 5 & 131041 & 14 & 14004 & 5 & 132312 & 111 & 1242 \\
\hline 5 & 124421 & 11 & 11311 & 5 & 131112 & & & 5 & 132314 & 141 & 1404 \\
\hline 5 & 124423 & & & 5 & 131114 & 11 & 12424 & 5 & 132323 & 13 & 10221 \\
\hline 5 & 124424 & 13 & 14233 & 5 & 131123 & & & 5 & 132324 & 11 & 12034 \\
\hline 5 & 124431 & 14 & 13214 & 5 & 131124 & 13 & 10133 & 5 & 132331 & 13 & 10222 \\
\hline 5 & 124432 & 11 & 11312 & 5 & 131131 & 11 & 12421 & 5 & 132332 & & \\
\hline
\end{tabular}


Factor Tables

Polynomials, Modulo 5-Continued

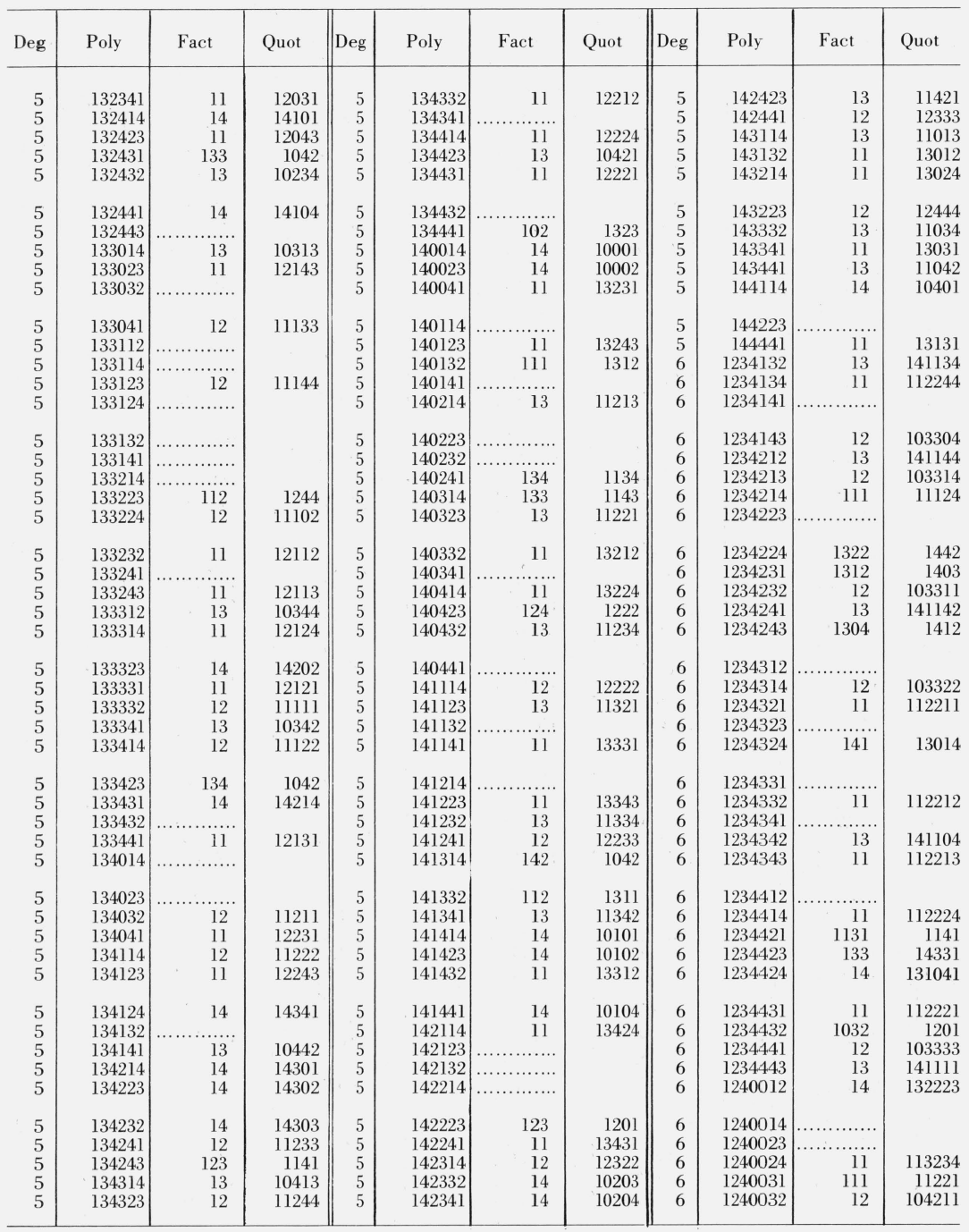


FACTOR TABLES

Polynomials, Modulo 5-Continued

\begin{tabular}{|c|c|c|c|c|c|c|c|c|c|c|c|}
\hline Deg & Poly & Fact & Quot & Deg & Poly & Fact & Quot & Deg & Poly & Fact & Quot \\
\hline 6 & 1240041 & 11 & 113231 & 7 & 11211341 & 12 & 1443033 & 7 & 11212221 & 111 & 101011 \\
\hline 6 & 1240043 & 142 & 13044 & 7 & 11211342 & 14 & 1240143 & 7 & 11212223 & 11 & 1024343 \\
\hline 6 & 1240112 & 11 & 113242 & 7 & 11211343 & 11 & 1024213 & 7 & 11212224 & 12 & 1443102 \\
\hline 6 & 1240114 & 12 & 104222 & 7 & 11211344 & 1113 & 10123 & 7 & 11212231 & 12 & 1443103 \\
\hline 6 & 1240123 & 11 & 113243 & 7 & 11211411 & 12 & 1443043 & 7 & 11212232 & 142 & 122421 \\
\hline 6 & 1240124 & 102 & 12212 & 7 & 11211412 & 13 & 1332044 & 7 & 11212233 & 13 & 1332141 \\
\hline 6 & 1240131 & 123 & 10132 & 7 & 11211413 & & & 7 & 11212234 & 11 & 1024344 \\
\hline 6 & 1240132 & 1311 & 1412 & 7 & 11211414 & 11 & 1024224 & 7 & 11212241 & 13 & 1332142 \\
\hline 6 & 1240134 & 11 & 113244 & 7 & 11211421 & & & 7 & 11212242 & 14 & 1240243 \\
\hline 6 & 1240141 & 13 & 142442 & 7 & 11211422 & 102 & 110411 & 7 & 11212243 & 12 & 1443104 \\
\hline 6 & 1240143 & 14 & 132232 & 7 & 11211423 & 12 & 1443044 & 7 & 11212244 & & \\
\hline 6 & 1240212 & & & 7 & 11211424 & 112 & 100102 & 7 & 11212311 & 11 & 1024301 \\
\hline 6 & 1240213 & 13 & 142401 & 7 & 11211431 & 11 & 1024221 & 8 & 114313414 & 134 & 1313321 \\
\hline 6 & 1240214 & & & 7 & 11211432 & 14 & 1240103 & 8 & 114313421 & 14 & 12140324 \\
\hline 6 & 1240223 & 1024 & 1222 & 7 & 11211433 & 13 & 1332041 & 8 & 114313423 & 12 & 14114044 \\
\hline 6 & 1240224 & 14 & 132241 & 7 & 11211434 & & & 8 & 114313424 & 11 & 10442134 \\
\hline 6 & 1240231 & 1101 & 1131 & 7 & 11211441 & 13 & 1332042 & 8 & 114313431 & 141 & 1201241 \\
\hline 6 & 1240232 & 1312 & 1411 & 7 & 11211442 & 11 & 1024222 & 8 & 114313432 & 13 & 13032234 \\
\hline 6 & 1240241 & 12 & 104233 & 7 & 11211443 & 1444 & 12042 & 8 & 114313434 & 1032 & 111312 \\
\hline 6 & 1240243 & 124 & 10002 & 7 & 11212012 & 14 & 1240223 & 8 & 114313441 & 11 & 10442131 \\
\hline 6 & 1240312 & 124 & 10003 & 7 & 11212013 & 133 & 130211 & 8 & 114313442 & 12 & 14114041 \\
\hline 6 & 1240314 & 13 & 142413 & 7 & 11212014 & 11 & 1024324 & 8 & 114313443 & & \\
\hline 6 & 1240323 & 12 & 104244 & 7 & 11212021 & 14 & 1240224 & 8 & 114314012 & & \\
\hline 6 & 1240324 & 133 & 14413 & 7 & 11212023 & 13 & 1332121 & 8 & 114314013 & 11 & 10442233 \\
\hline 6 & 1240331 & 1141 & 1141 & 7 & 11212024 & 141 & 123214 & 8 & 114314014 & 13 & 13032313 \\
\hline 6 & 1240332 & 11 & 113212 & 7 & 11212031 & 11 & 1024321 & 8 & 114314021 & 111 & 1030311 \\
\hline 6 & 1240341 & 14 & 132204 & 7 & 11212032 & 123 & 141204 & 8 & 114314023 & 103 & 1110341 \\
\hline 6 & 1240343 & 11 & 113213 & 7 & 11212034 & 12 & 1443132 & 8 & 114314024 & 11 & 10442234 \\
\hline 6 & 1240412 & 12 & 104201 & 7 & 11212041 & 12 & 1443133 & 8 & 114314031 & & \\
\hline 6 & 1240414 & 11 & 113224 & 7 & 11212042 & 11 & 1024322 & 8 & 114314032 & 123 & 1430204 \\
\hline 6 & 1240421 & 141 & 13131 & 7 & 11212043 & & & 8 & 114314034 & 12 & 14114132 \\
\hline 6 & 1240423 & 13 & 142421 & 7 & 11212112 & 1223 & 14214 & 8 & 114314041 & 11 & 10442231 \\
\hline 6 & 1240424 & 12 & 104202 & 7 & 11212113 & 11 & 1024333 & 8 & 114314042 & 14 & 12140443 \\
\hline 6 & 1240431 & 11 & 113221 & 7 & 11212114 & & & 8 & 114314043 & 13 & 13032311 \\
\hline 6 & 1240432 & 1323 & 1404 & 7 & 11212121 & 1042 & 11303 & 8 & 114314112 & 11 & 10442242 \\
\hline 6 & 1240441 & & & 7 & 11212122 & & & 8 & 114314113 & 124 & 1423232 \\
\hline 6 & 1240443 & 12 & 104204 & 7 & 11212123 & 12 & 1443144 & 8 & 114314114 & 14 & 12140401 \\
\hline 7 & 11211243 & 14 & 1240132 & 7 & 11212124 & 11 & 1024334 & 8 & 114314121 & 103 & 1110342 \\
\hline 7 & 11211244 & 11 & 1024204 & 7 & 11212131 & & & 8 & 114314123 & 11 & 10442243 \\
\hline 7 & 11211311 & 13 & 1332032 & 7 & 11212132 & 13 & 1332134 & 8 & 114314124 & & \\
\hline 7 & 11211312 & 112 & 100101 & 7 & 11212133 & & & 8 & 114314131 & 13 & 13032322 \\
\hline 7 & 11211313 & & & 7 & 11212134 & 14 & 1240231 & 8 & 114314132 & 14 & 12140403 \\
\hline 7 & 11211314 & 124 & 140011 & 7 & 11212141 & 11 & 1024331 & 8 & 114314134 & 11 & 10442244 \\
\hline 7 & 11211321 & 11 & 1024211 & 7 & 11212142 & 12 & 1443141 & 8 & 114314141 & 14 & 12140404 \\
\hline 7 & 11211322 & 12 & 1443031 & 7 & 11212143 & 14 & 1240232 & 8 & 114314142 & 12 & 14114141 \\
\hline 7 & 11211323 & & & 7 & 11212144 & & & 8 & 114314143 & 1244 & 142442 \\
\hline 7 & 11211324 & 13 & 1332033 & 7 & 11212211 & 1033 & 11402 & 8 & 114314212 & 12 & 14114101 \\
\hline 7 & 11211331 & 1312 & 13203 & 7 & 11212212 & 11 & 1024342 & 8 & 114314213 & 14 & 12140412 \\
\hline 7 & 11211332 & 11 & 1024212 & 7 & 11212213 & 112 & 100114 & 8 & 114314214 & 10123 & 11303 \\
\hline 7 & 11211334 & 12 & 1443032 & 7 & 11212214 & & & 8 & 114314221 & 1341 & 131231 \\
\hline
\end{tabular}


Polynomials, Modulo 5-Continued

\begin{tabular}{|c|c|c|c|c|c|c|c|c|c|c|c|}
\hline Deg & Poly & Fact & Quot & Deg & Poly & Fact & Quot & Deg & Poly & Fact & Quot \\
\hline 8 & 114314223 & 123 & 1430201 & 8 & 140144232 & 1222 & 124231 & 8 & 140201323 & & \\
\hline 8 & 1143 & 12 & 14114102 & 8 & 140 & 11 & 13240431 & 8 & 1332 & 12 & 12100111 \\
\hline 8 & 114314231 & 12 & 14114103 & 8 & 140144314 & 102 & 1433332 & 8 & 140201341 & 1322 & 110343 \\
\hline 8 & 114314232 & 13 & 13032334 & 8 & 140144323 & 11 & 13240443 & 8 & 140201414 & 12 & 12100122 \\
\hline 8 & 114314233 & 11 & 10442203 & 8 & 140144332 & & & 8 & 140201423 & 124 & 1220222 \\
\hline 8 & 14314234 & & & 8 & 140144341 & & & 8 & 140201432 & & \\
\hline 8 & 1143 & 102 & 1121223 & 8 & 140 & & & 8 & 140201441 & 11 & 13200131 \\
\hline 8 & 114314242 & 10102 & 11321 & 8 & 140144423 & 1032 & 142204 & 8 & 140202014 & 103 & 1420423 \\
\hline 8 & 114314243 & 12 & 14114104 & 8 & 140144432 & 13 & 11204234 & 8 & 140202023 & 13 & 11212121 \\
\hline 8 & 114314312 & 13 & 13032344 & 8 & 140144441 & & & 8 & 140202041 & 11 & 13200231 \\
\hline 8 & 114314313 & 12 & 14114114 & 8 & 140200114 & 124 & 1220211 & 8 & 140202114 & 12 & 12100222 \\
\hline 8 & 11431 & & & 8 & 0123 & 112 & 1301 & 8 & & 11 & 13200243 \\
\hline 8 & 1143 & 11 & 10442211 & 8 & 140200132 & 11 & 13200 & 8 & 140 & 13 & 11212134 \\
\hline 8 & 114314323 & & & 8 & 140200141 & 13 & 11212442 & 8 & 140202141 & 14 & 10022404 \\
\hline 8 & 114314324 & 1201 & 141024 & 8 & 140200214 & 11 & 13200024 & 8 & 140202214 & 12021 & 12134 \\
\hline 8 & 114 & 1322 & 133113 & 8 & 140 & & & 8 & & 12312 & 12344 \\
\hline 8 & 1143 & 11 & 10442212 & 8 & 140 & & & 8 & 232 & 123 & 1230104 \\
\hline 8 & 114314334 & 1134 & 101301 & 8 & 140200241 & 1214 & 120104 & 8 & 140202241 & 12 & 12100233 \\
\hline 8 & 114314341 & 13 & 13032342 & 8 & 1402 & 13 & 11212413 & 8 & 140202314 & & \\
\hline 8 & 114314342 & & & 8 & 140200323 & 14 & 10022202 & 8 & 140202323 & 12 & 12100244 \\
\hline 8 & 1 & 11 & 1 & 8 & & 14 & & 8 & & 11 & 13200212 \\
\hline 8 & $114:$ & 14 & 1214 & 8 & i & 11 & 132 & 8 & & & \\
\hline 8 & 1143 & 134 & 131 & 8 & & 1011 & & 8 & & 11 & 13200224 \\
\hline 8 & 114314413 & 13 & 13032301 & 8 & 140 & 11 & 132 & 8 & & 123 & 1230101 \\
\hline 8 & 114314414 & 11 & 10442224 & 8 & 140200432 & 111 & 1313112 & 8 & 140202432 & 102 & 1434441 \\
\hline 8 & 114 & 12 & 14114123 & 8 & 14 & & & 8 & & 11222 & 13043 \\
\hline 8 & 114 & & & 8 & & 102 & 1434432 & 8 & & 11 & 13200324 \\
\hline 8 & 1143 & 12021 & 4 & 8 & 140 & 11 & 13200143 & 8 & 144 & 14 & 10022003 \\
\hline 8 & 1143 & 1 & 1044 & 8 & & & & 8 & & 13 & 11212242 \\
\hline 8 & 114314432 & 112 & 1021111 & 8 & 140201114 & 13 & 11212013 & 8 & 140203114 & 1311 & 111224 \\
\hline 8 & 114 & 13 & 13032303 & 8 & 14 & 12 & 121 & 8 & & 11113 & 13 \\
\hline 8 & 1143 & & & 8 & 141 & 103 & 1420414 & 8 & 132 & 1014 & 144403 \\
\hline 8 & 1143 & 11 & 104 & 8 & & 10313 & & 8 & & 11 & 13200331 \\
\hline 8 & 1143 & 14 & 1214 & 8 & & 14 & 10022301 & 8 & 140 & 13 & 11212213 \\
\hline 8 & 114320012 & 12 & 14110001 & 8 & 140201223 & 13 & 11212021 & 8 & 140203223 & 11 & 13200343 \\
\hline 8 & 1143 & 11 & 1044.3 & 8 & & 11 & 13200112 & 8 & 140203232 & 12 & 12100311 \\
\hline 8 & 1143 & 102 & & 8 & & 14 & & & & & \\
\hline 8 & 140144223 & 11402 & 13314 & 8 & 140201314 & 11 & 13200124 & & & & \\
\hline
\end{tabular}

\section{References}

[1] Bauer, M., Journal de Crelle, 128, 298, (1905).

[2] Dorwart, H. L. Irreducibility of Polynomials, Amer. Math. Monthly 42, 369-381, (1935).

[3] Dumas, G. Journal de Mathematique, 191, (1906).

[4] Eisenstein, G., Uber die Irreductibilitat und andere Eisenschaften der Gleichung, Journal fur die reine und angewandte Mathematik 39, 166-179, (1850).

[5] Frumveller, A. F., Quadratic Factors of Polynomials, Amer. Math. Monthly 24, 208-212, (1917).

[6] Glenn, O. E., Relating to the quadratic factors of a polynomial, Amer. Math. Monthly 23, 313-315, (1916).

[7] Königsberger, Journal fur Mathematik 115, 53, (1895).

[8] Kronecker, L., Grundzuge einer arithmetischen Theorie der Algebraischen Grossen, Journal fur die reine und angewandte Mathematik 92, 111-114.

[9] Lloyd, D. B.. Some Properties of Rational Quintic Equations University Press, (1940).

[10] Lloyd, D. B., Factorization of the general polynomial by means of its homomorphic congruential functions, Am. Math'l. Monthly 71, 8, (1964).
[11] Mandl, M., Uber die Zerlegung ganzer, ganzzahliger Functionen in irreductible Factoren, Journal fur die reine und angewandte Mathematik 113, 252-261, (1894).

[12] Netto, Mathematische Annalen 48, 81, (1897).

[13] Ore, O., Mathematische Zeitschrift 18, 278, (1893); 20, 267, (1924).

[14] Perron, Mathematische Annalen 60, 448, (1905).

[15] Peterson, W. W., Error-Correction Codes (John Wiley \& Sons, New York, N.Y., 1961.

[16] Polya, G., and Szego, Aufgaben und Lehrsatze aus der Analysis, II, 350 .

[17] Runge, Irreductibilitat der Gleichung, Journal die reine und angew andte Mathematik 99, 89-97, (1886).

[18] Schoenemann, Journal fur Mathematik 32, 100, (1846).

[19] Stäckel, Journal fur Mathematik 148, 101, (1918).

[20] Varnhorn, M. C., Some Properties of Quartic Functions of One Variable (Catholic University Press, Washington, D.C., 1939). 